\title{
Synthesis and Screening of \\ Phase Change Chalcogenide Thin Film Materials for Data Storage
}

Samuel Guerin ${ }^{1}$, Brian Hayden ${ }^{1,2}$ *, Daniel W Hewak ${ }^{3}$ and Chris. Vian ${ }^{1}$

1. Ilika Technologies, Kenneth Dibben House, University of Southampton Science Park, Chilworth, Southampton, S016 7NS, UK.

2. Chemistry, University of Southampton, Southampton, S017 1BJ, UK.

3. Optoelectronics Research Centre, University of Southampton, Southampton, S017 1BJ, UK.

\section{ABSTRACT}

A combinatorial synthetic methodology based on evaporation sources in ultra-high vacuum has been used to directly synthesise compositional gradient thin film libraries of the amorphous phases of GeSbTe alloys at room temperature over a wide compositional range. An optical screen is described which allows the rapid parallel mapping of the amorphous to crystalline phase transition temperature, and the optical contrast associated with the phase change, on such libraries. The results are shown to be consistent with the literature for compositions where published data are available along the $\mathrm{Sb}_{2} \mathrm{Te}_{3}-\mathrm{GeTe}$ tie line. The results reveal a minimum in the crystallisation temperature along the $\mathrm{Sb}_{2} \mathrm{Te}_{3}-\mathrm{Ge}_{2} \mathrm{Te}_{3}$ tie line, and the method is able to resolve subsequent cubic to hexagonal phase transitions in the GST crystalline phase. HT-XRD has been used to map the phases at sequentially higher temperatures and the results are reconciled with the literature and trends in crystallisation temperatures. The results clearly delineate compositions which crystallise to pure GST phases, and those which co-crystallise Te. High throughput measurement of the resistivity of the 
amorphous and crystalline phases has allowed the compositional and structural correlation of the resistivity contrast associated with the amorphous to crystalline transition which range from 5 to 8 orders of magnitude for the compositions investigated. The results are discussed in terms of the compromises in the selection of these materials for phase change memory applications, and the potential for further exploration through more detailed secondary screening of doped GST or similar classes of phase change materials designed for the demands of future memory devices.

Keywords: High-Throughput, PCM, GST, HTOMPT

* Corresponding Author 


\section{INTRODUCTION}

Chalcogenide materials for data storage applications, both for optical storage media ${ }^{1}$ or the next generation of RAM and Flash ${ }^{\circledR}$ memory applications ${ }^{2}$, rely on the thermally induced phase change between the amorphous and polycrystalline state in a small volume of the material. Characterisation of the basic amorphous to crystalline phase changes in new ternary or quaternary materials is a prerequisite for the further screening and optimisation of the material for the various storage configurations. This will require the determination of figures of merit for resistivity, electrical switching characteristics, time responses or reflectivity change and optical absorption characteristics depending on the specific application or technology. These parameters are critical for optimizing switching speed and power consumption. The need for high data density means that the properties of very small volumes are crucial, hence favouring studies of thin films of a similar thickness to real device applications as a primary screen for establishing their intrinsic properties. We present here a combinatorial synthesis and an associated primary high throughput screening methodology for rapid identification of promising compositions. We also briefly describe the associated characterisation methods and highlight the potential of the synthetic method for further primary and secondary screening, comparing it to approaches previously applied to sputtered materials ${ }^{3-4}$.

In the past, amorphous compositions of chalcogenide materials have been investigated through the sequential synthesis of the bulk material by conventional glass melting techniques; sealing the constituent elements in ampoules, heating and homogenizing and subsequently quenching to produce the glass ${ }^{5}$. Typically, differential thermal analysis (DTA) combined with X-ray diffraction allows identification of the phase transition. There are many properties which must be optimised to synthesise a useful phase change material, while at the same time significant changes in performance can be observed with small compositional variations. However, chalcogenide-based phase change materials lend themselves to high-throughput synthesis and screening through a 
compositional spread approach that is applied to thin films of the material. In the past, compositional ranges of GeSbTe synthesised using multiple target sputtering onto a $\mathrm{SiO}_{2}$ on $\mathrm{Si}$ substrate were limited to relatively small compositional ranges; within approximately 6 at. $\%$ of the well characterised $\mathrm{Ge}_{2} \mathrm{Sb}_{2} \mathrm{Te}_{5}$ eutectic composition ${ }^{3}$ and more recently to around $10 \%$ variation for four compositions of interest along the $\mathrm{Sb}_{2} \mathrm{Te}_{3}$-GeTe tie line ${ }^{4}$. The experiment though limited in the compositional space explored, demonstrated the efficiency of high throughput secondary (sequential) screens ${ }^{6}$ to characterise the write/erase time and crystal structure dependence on composition. More recently, sequential deposition using a shutter system to periodically deposit thin films of various elements to create a layered structure has been used to synthesise GeSbTe thin films ${ }^{7}$. The material was subsequently annealed to allow the constituent atoms to mix and produce the crystalline alloy phases.

We have applied a synthesis based on a high throughput physical deposition (HT-PVD) method 8 which can produce high purity, equilibrated and non-equilibrated solid state thin film libraries. The method allows the simultaneous and controlled co-deposition of the atomic elements on a substrate through evaporation to yield a pre-determined wide or narrow compositional spread of material as a function of array position. For each location, the resulting composition is the normalised sum of the constituent elements co-evaporated simultaneously for a given time. The composition of GeSbTe only varies as a function of $(\mathrm{x}, \mathrm{y})$ position and is constant through the thickness of the film. We have used the synthesis to directly produce the amorphous phases of the ternary system through deposition on a substrate at room temperature. In order to demonstrate the synthetic method and subsequent primary screen, we present results for a wide compositional spread, covering 50 to $55 \%$ of the phase diagram, of the ternary GeSbTe chalcogenide alloy system which has been investigated extensively because of its use in optical data storage applications ${ }^{9-12}$. A set of fundamental experiments comprising of optical reflectivity, X-ray diffraction and conductivity as a function of, both, temperature 
and composition has been carried out on the thin films and forms the basis of the primary screen of thin films within this compositional space. For this system, we have characterised the optical changes of the thin films as they are annealed from room temperature to $400{ }^{\circ} \mathrm{C}$, linked these changes to the amorphous to crystalline phase transition, and in so doing generate compositional maps of the crystalline transition temperatures $\left(T_{c}\right)$ and the magnitude of the normalised changes in reflectivity $\left(\Delta R / R_{0}\right)$. The importance of $T_{c}$ in a primary screen is that this figure of merit reflects the balance between the potential stability of the amorphous phase (and hence the robustness and longevity of data storage) and the potential power requirements for switching. In addition, automated XRD studies for thin films in the as deposited state and after annealing at $150^{\circ} \mathrm{C}, 200^{\circ} \mathrm{C}$ and $300^{\circ} \mathrm{C}$ are shown providing direct evidence of crystallisation and phase mapping of the crystalline phases. Finally, resistivity studies for thin films in the as-deposited (amorphous) and annealed (crystalline) state are also presented. The optical $\left(\Delta R / R_{0}\right)$ and electrical $\left(\Delta R_{E}\right)$ contrast associated with the phase change reflects the potential dynamic range accessible for data storage, and the possibility of storing multiple bits through partial phase change. The data sets produced from this primary screening of materials is intended to provide the basis for the selection of compositions which will exhibit the switching contrast necessary for effective storage, remain stable at operating temperatures without the need to excessive powers for switching or reset, and will remain stable during operation without phase separation. The GeSbTe compositional spread investigated includes those materials that have been extensively characterised and reported in the literature to validate the approach, and is extended to a wider range of compositions.

\section{EXPERIMENTAL}

Details of the HT-PVD system and the synthetic method used to produce compositionally varying thin film materials has been described in detail elsewhere ${ }^{8}$. In the current work, experiments have been carried out in an ultra-high vacuum (UHV) system (DCA Instruments), base pressure 1×10-10 mBar 
and consisting of two cryo-pumped PVD chambers, an ion pumped surface analysis and reaction chamber, and a turbo-molecularly pumped RF sputtering chamber, masking station and ion pumped interconnect lines allowing transfer of substrates between chambers. The Sb and Te (both 99.999\% from ABSCO Materials) were evaporated from Knudsen cells and the Ge $(99.999 \%$ from ABSCO Materials) was evaporated from an electron gun (Temescal model SFIH-270-2). The thin films were deposited on $\mathrm{Si}<100>$ for composition determination, for XRD measurements and for HTOMPT (see below) optical reflectivity measurements. SiN on $\mathrm{Si}<100>$ or silica substrates were used for XRD, 4PP or HTOMPT measurements (all from NOVA Electronics). The substrate footprints were $32 \times 32$ $\mathrm{mm}^{2}$. Prior to deposition, the substrates were cleaned in an ultrasonic bath for $10 \mathrm{~min}$ in $50 \%$ solution of ethanol absolute in deionized water and $10 \mathrm{~min}$ in iso-propanol absolute. Clean substrates were dried in a flow of nitrogen.

The thin film deposited were either continuous films of dimensions $28 \times 28 \mathrm{~mm}^{2}$ centred on the substrate or (for optical profilometry measurement of thickness) on an array of discreet fields using a shadow mask; In the case of the discreet fields, we have used a matrix of $10 \times 10$ fields each of $1.2 \times 1.2 \mathrm{~mm}^{2}$ and with a pitch between fields of $2 \mathrm{~mm}$. In the case of continuous films, the analyses were done using either a $12 \times 12$ matrix with a $2 \mathrm{~mm}$ pitch, or a $23 \times 23$ matrix with a $1 \mathrm{~mm}$. In both cases this covers an area of $22 \times 22 \mathrm{~mm}^{2}$.

The compositional spread covered by each sample was measured by Energy Dispersive Spectroscopy using an Oxford Instruments EDS system on a JEOL JSM-5910 SEM with an automated stage to allow for programmable analysis of the sample. A $12 \times 12$ macro with a pitch of $2 \mathrm{~mm}$ between fields was used. This $12 \times 12$ was interpolated to a physically equivalent $23 \times 23$ array with a pitch between fields of $1 \mathrm{~mm}$ to map the resistivity and XRD data. For a given compositional spread investigated several samples (referred as a set) were synthesised to be able to carry out all the necessary measurements. 
The synthesis conditions for each sample within a set were kept identical and EDS was performed on the first and the last sample of each set in order to ensure the desired compositional spread was covered.

A sample with discreet fields was prepared within each set under identical conditions to those used for the rest of the set. This sample was subsequently covered by a uniform layer of Sb, this providing a layer of equivalent optical properties across the whole sample. The thickness was then measured on a Veeco WYKO NT-1100 optical profilometer with a vertical resolution of $0.3 \mathrm{~nm}$ using an automated macro. Due to the array dimensions described above, the resulting thickness map corresponded to the $18 \times 18 \mathrm{~mm}^{2}$ area and needed to be extrapolated to the larger $22 \times 22 \mathrm{~mm}^{2}$ area of the continuous film to have thicknesses for this area which could be used to calculate the resistivity of the thin films. The extrapolation was done using a quadratic function. The data was subsequently interpolated to convert the $12 \times 12$ into a $23 \times 23$ matrix with a $1 \mathrm{~mm}$ pitch to match the XRD and resistivity data.

A purpose-built apparatus (see supplementary information) was developed to measure changes in optical reflectivity in real time on a compositional spread thin film sample as a function of temperature. In the case of phase change memory materials (and indeed a range of other amorphous materials and glasses ${ }^{13}$ ) there is a direct link between the changes in reflectivity and changes in the phase of the material, hence the associated acronym HTOMPT (High Throughput Optical Mapping of Phase Transition) to describe the method. An inert Ar atmosphere is used to protect the sample during the heating ramp. The apparatus consists of a copper block on which the sample is mounted, with two embedded cartridge heaters operated via a Eurotherm 3508 controller; the heating rate used in this work was $5^{\circ} \mathrm{C} \mathrm{min-1}$. The whole block assembly is placed inside a sealed stainless steel chamber with a top window. Uniform white lighting is provided by a halogen lamp and a light diffuser, 
an off-axis CCD camera (Lumenera Infinity 2-1M) is used to record images of the sample at set time intervals of $12 \mathrm{~s}$ thus providing one picture per degree. The highest spatial resolution achieved over the sample library, and hence the compositional resolution of the measurement, is determined by the number of pixels $(1392 \times 1040)$ of the image frame. In the present study we have averaged the intensities of the pixels to increase the signal to noise of the reflectivity measurement, measuring at an effective resolution of a $120 \times 120$ matrix over the $22 \times 22 \mathrm{~mm}^{2}$ area of the library.

X-ray diffraction was performed after synthesis on the $23 \times 23$ matrix , and following annealing to $150^{\circ} \mathrm{C}, 200^{\circ} \mathrm{C}$ and $300^{\circ} \mathrm{C}$ using a D8 Bruker diffractometer equipped with a GADDS detector and an Incoatec I IS X-ray source (Cu $1.54059 \AA$ ) with an X-ray beam of $0.25 \mathrm{~mm}$ in diameter. The incident angle used was $11^{\circ}$ giving an elongated beam size in the diffraction plane at the sample of maximum $1.31 \mathrm{~mm}$, and $0.25 \mathrm{~mm}$ in the direction orthogonal to the plane. The sample used for this measurement was deposited on $\mathrm{SiO}_{2}$.

Resistivity of the thin films was obtained by combining the sheet resistance measurement with the thickness measurements. The sheet resistance was measured using a $23 \times 23$ matrix on a Signatone probe station equipped for 4-point probe measurement using two Keithley 2636 SourceMeters. The sheet resistance was measured on the samples deposited on $\mathrm{SiO}_{2}$ substrates in the as-deposited state and after annealing to $200^{\circ} \mathrm{C}, 300^{\circ} \mathrm{C}$ and $400^{\circ} \mathrm{C}$.

\section{RESULTS AND DISCUSSION}


Figure 1 shows the compositional spread of a single GeSbTe thin film library measured by energy dispersive X-ray spectroscopy (EDS) on a 12×12 matrix. Elemental distributions of single elements are shown as relative atomic percentages in colour maps of the actual deposited library (Figure 1A), and in a ternary compositional plot (Figure 1B). The overall compositional range of this library covers 10-86 at\% Te, 4-72 at\% Ge and 0-70 at\% Sb. The compositional spread of a thin film library can be varied considerably and reproducibly on a single substrate by optimising the deposition rate of the elements and the appropriate setting of the "wedge" shutters ${ }^{8}$. In this case the region of compositions within the library to covers a large part of the ternary compositions of possible GeSbTe phases, a strategy appropriate for primary screening of key figures of merit. For comparison, the previously published high throughput depositions made using multiple sputtering targets are indicated by the shaded areas in Figure 1B ${ }^{4}$. The points of composition measured by EDS do not represent the compositional increments that can potentially be screened, they only represent the envelope of material compositions synthesised. Rather the screening measurement applied to a $28 \times 28 \mathrm{~mm}$ area of library determines the compositional resolution which can be achieved for the corresponding figure of merit. Due to the nature of the deposition method ${ }^{8}$ the compositions vary smoothly over the sample (elemental contour maps in Figure 1A), the EDS data can be confidently interpolated in order to yield a composition for a higher resolution screening measurement on a finer matrix. However, calculating relative atomic percentages of relatively thin films $(<100 \mathrm{~nm})$ can lead to an increase in the standard deviations observed. This can cause larger variations in compositions, especially when interpolating a $12 \times 12$ library to a higher number such as the $23 \times 23$ and $120 \times 120$ used in the present work. To improve on compositional accuracy, the calculated $12 \times 12$ compositional data was smoothed by applying a weighted nearest neighbour averaging for each elemental $12 \times 12$ matrix. Then the EDX data was interpolated from a $12 \times 12$ matrix to a $23 \times 23$ matrix and a $120 \times 120$ matrix. The $23 \times 23$ matrix, which corresponds to a pitch between measurements of ca.1 mm, 
provides a reliable composition for the XRD and the resistivity measurements: These have been carried out on a finer matrix with measurement points separated by ca. $1 \mathrm{~mm}$ appropriate to the probes spatial resolution. The $120 \times 120$ matrix was used for the analysis of the optical data.

Figure $1 \mathrm{C}$ shows the thickness distribution as a function of composition across the library. The minimum thickness is $50 \mathrm{~nm}$ for $\mathrm{Ge}_{4} \mathrm{Sb}_{41} \mathrm{Te}_{55}$, and the maximum is $224 \mathrm{~nm}$ for $\mathrm{Ge}_{12} \mathrm{Te}_{88}$. The variations in thickness are due to the synthesis method and are inherent to the use of three off axis source with our wedge growth method ${ }^{8}$.

The crystallisation temperature $\left(T_{c}\right)$ and the relative change in optical reflectivity $(\Delta R / R)$ associated with crystallisation were investigated using HTOMPT. Reflectivity changes ${ }^{14-15}$ (and indeed changes in resistivity ${ }^{16}$ ) associated with a phase transformation when driven by a linear ramp in temperature typically exhibit a sigmoidal function. The inflection of the sigmoidal curve corresponds, for the amorphous/crystalline transition, to the temperature of the fastest rate of crystallisation at the applied heating rate. For a process of homogeneous nucleation and growth under thermal equilibrium, the temperature of this maximum crystallisation rate is proportional to, and very close to (for practical heating rates) the crystallisation temperature $T_{c}$. Similar curves for glass transitions are traditionally obtained in differential scanning calorimetry (DSC) or differential thermal analysis (DTA) where the maximum in the rate of crystallisation is associated with the peak maximum in the derivative of the DTA curve (DDTA) ${ }^{17}$. This approach has been applied previously for single composition thin films of GeSbTe of comparing Tc for various GeSbTe compositions ${ }^{15}$. In the HTOMPT measurement, each pixel of the image corresponds to the intensity of the reflected light at a defined composition of an initially amorphous thin film. To extract the inflection point associated with the transition (and hence Tc) during the non-isothermal measurement, the measured reflected intensity (I) is normalised to the intensity of reflected light ( $\left.I_{0}\right)$ in the initial amorphous phase, since each 
amorphous composition reflects the light differently. Practically these values of reflected intensity are averages over a defined number of pixels corresponding to a finite (and small) compositional variation in the film. Examples of the measured curves $\left(\mathrm{l} / \mathrm{I}_{0}\right)$ for some fixed compositions can be found in Figure 2B. In these experiments, the temperature of the compositional gradient thin film library was increased from room temperature to $400^{\circ} \mathrm{C}$ at a linear heating rate of $5^{\circ} \mathrm{C} \mathrm{min}-1$. The sample was protected from thermally induced oxidation / reaction using a constant flow of Ar over the sample. Images were recorded at 12 s time intervals, corresponding to a reflectivity measurement every $1^{\circ} \mathrm{C}$. The recorded film at an accelerated speed of $25 \mathrm{x}$ for a library is shown in the Supplementary Material. The time dependent images are processed to extract, in the first instance, the relative change in optical reflectivity $\left(\mathrm{I}-\mathrm{I}_{0}\right)$ in a $120 \times 120$ matrix of compositions over the $22 \times 22$ $\mathrm{mm}^{2}$ area of the library. The HTOMPT data relies on a direct correlation between the changes in optical reflectivity and changes in the material's phase or crystal structure. In the case of chalcogenide alloys, such a direct correlation is well established and forms the basis of optical data storage ${ }^{18}$. Furthermore, XRD data obtained on our libraries at various temperatures confirmed that the initial increases in reflectivity (and sigmoidal curve) were associated with the amorphous/crystalline phase change. Note that there is consistently a decrease in reflectivity observed at higher temperatures following crystallisation: We associate this with morphological changes (or for some compositions, compositional changes) in the thin film above ca. $350^{\circ} \mathrm{C}$.

The time lapse images from HTOMPT of the non-isothermal crystallisation measurements are used to extract $T_{c}$ as a function of composition (Figure $2 A$ ). The transition temperature $T_{c}$ was calculated from the sigmoidal temperature dependence curve $\left(\mathrm{I}-\mathrm{I}_{0}\right)$ using a derivative method. Some examples of the reflectivity changes $\left(\mathrm{I}-\mathrm{I}_{0}\right)$ and its first derivative versus temperature are shown in Figure $2 \mathrm{C}$. The first and second derivatives of the data were calculated. The identification of a phase transition, and the associated maximum rate of crystallisation (and hence $T_{c}$ ) was achieved by setting the 
condition that the first derivative had to cross a threshold value, and the second derivative had to become zero. This approach works well for identifying the first transition (amorphous to first crystalline state) in a completely automated manner. In GeSbTe, a second transition is often observed, and usually associated with a cubic to hexagonal phase transition ${ }^{19}$, identifying it using an automated algorithm proved difficult to implement as the magnitude, duration and position of the second transition with respect to the first transition was too variable. For this second transition, to date, only a manual analysis of the data leads to a correct estimate of the second transition temperature.

The optical contrast associated with full crystallisation (to the highest temperature) was also extracted from the time lapse images of the non-isothermal crystallisation measurements, and this compositional dependence is shown in Figure 3. Complete crystallisation (before any temperature induced morphological changes) are determined from the change in reflected intensity between the amorphous material $\left(I_{0}\right)$ and the fully crystallised materials $I_{c}$ (at the maximum in the reflected intensity curve), and normalised to the reflectivity of the amorphous material i.e. $\left(I_{c}-I_{0}\right) / I_{0}$ : This optical contrast is expressed in Figure 3 as a percentage. Following crystallisation, a large region of the compositional space, broadly defined by the quadrilateral delimited by $\mathrm{Sb}_{30} \mathrm{Te}_{70}-\mathrm{Ge}_{30} \mathrm{Te}_{70}-$ $\mathrm{Ge}_{60} \mathrm{Te}_{40}-\mathrm{Sb}_{80} \mathrm{Te}_{20}$, exhibit large changes in optical contrast $\left.\left(\mathrm{I}_{\mathrm{c}}-\mathrm{I}_{0}\right) / \mathrm{I}_{0}>50 \%\right)$. The maximum change observed ( $85 \%$ ) has been found for the antimony poor compositions around $\mathrm{Ge}_{33} \mathrm{Sb}_{10} \mathrm{Te}_{57}$ in this library, with the composition of the most commercially exploited alloy $\mathrm{Ge}_{2} \mathrm{Sb}_{2} \mathrm{Te}_{5}$ exhibiting a change of $56.7 \%$. Along the tie-line $\mathrm{Sb}_{2} \mathrm{Te}_{3}-\mathrm{GeTe}$ the compositions $\mathrm{GeSb}_{4} \mathrm{Te}_{7}, \mathrm{GeSb}_{2} \mathrm{Te}_{4}$ and $\mathrm{Ge}_{4} \mathrm{SbTe}_{5}$ showed optical changes of $49.6,52.3$ and $71.9 \%$ respectively These values are consistent with the values reported in the literature which are expressed as a percentage increase in the intensity of the incident light $\left(I_{c}-I_{0}\right) / I_{\text {incident }}$; Typically the transition from the amorphous to crystalline phase is marked by an increase in $\left(I_{c}-I_{0}\right) / I_{\text {incident }}$ of around 20 to $30 \%{ }^{20-21}$. For example, Yamada et al. ${ }^{21}$ 
report $\mathrm{I}_{\mathrm{o}} / \mathrm{I}_{\text {incident }}$ of $8 \%$ ( amorphous state) and $\mathrm{I}_{\mathrm{c}} / \mathrm{I}_{\text {incident }}$ of $25 \%$ (crystalline state) corresponding to a normalised change in reflectivity $\left(I_{c}-I_{0}\right) / I_{0}$ of ca. $100 \%$. Similarly Gervacio Arciniera et al. ${ }^{15}$ would seem to indicate normalised change in reflectivity $\left(I_{c}-I_{0}\right) / I_{0}$ of 70 to $100 \%$ for the most common compositions along the $\mathrm{Sb}_{2} \mathrm{Te}_{3}-\mathrm{GeTe}$ tie-line. Our results are also consistent with the reflectivity changes associated with laser written marks of 500 microns diameter in a $50 \mathrm{~nm}$ thick film ${ }^{20}$. The high throughput synthetic and screening approach therefore allows a reliable and rapid comparisons of optical contrast available in optical storage applications: For the potential use in optical storage media, optical contrast would be only the first but necessary criteria for its suitability, and screens to determine the potential stability of phases, reversibility of transition and speed of the transition are also of importance, in addition to the stability of the phase change material within a composite structure.

From the ternary compositional dependence of $T_{C}$ (Figure $\left.2 A\right)$, the minimum value of $T_{C}$ was found to be $115^{\circ} \mathrm{C}$ for the $\mathrm{Ge}_{7} \mathrm{Sb}_{36} \mathrm{Te}_{58}$ composition, close to $\mathrm{Sb}_{2} \mathrm{Te}_{3}\left(\mathrm{~T}_{\mathrm{C}}=100{ }^{\circ} \mathrm{C}\right){ }^{15}$. Moving away from this composition the transition temperature gradually increases. The pseudo-binary tie-line joining GeTe to $\mathrm{Sb}_{2} \mathrm{Te}_{3}$ (indicated in Figure 2) has been traditionally extensively studied for two reasons: Firstly it links two stable binary alloys existing in the ternary space, and secondly its choice was driven by the fact that compositions along the tie line have a "facility for rapid crystallisation" 21 . Few studies have explored compositions outside the tie line; Krysta et al. ${ }^{3}$ have deposited, by sputtering, compositions around known metastable GST compounds but did not report crystallisation temperatures, whilst Yamada et al. ${ }^{21}$ indicate that the tie-line represents a valley of lowest crystallisation temperature (on the basis of a few points outside the tie line), with $\mathrm{T}_{\mathrm{C}}$ ranging from $100{ }^{\circ} \mathrm{C}$ to $150{ }^{\circ} \mathrm{C}$ for compositions between $\mathrm{Sb}_{2} \mathrm{Te}_{3}$ and $\mathrm{Ge}_{2} \mathrm{Sb}_{2} \mathrm{Te}_{5}$. In our study, we have found, along the $\mathrm{Sb}_{2} \mathrm{Te}_{3}-\mathrm{GeTe}$ line (within \pm 1 at\%) the Tc increasing from $117{ }^{\circ} \mathrm{C}$ to $168{ }^{\circ} \mathrm{C}$ for the compositions covered in this GeSbTe library. These crystallisation temperatures shown in Figure 2 have been projected onto the $\mathrm{Sb}_{2} \mathrm{Te}_{3}-\mathrm{GeTe}$ 
pseudo-binary tie line in Figure 4, and compared to the published data: The values of $T_{c}$ extracted from HTOMPT are, within experimental error, consistent with the literature ${ }^{22-24}$. Four stoichiometric compositions are often reported in the literature, three are referred as equilibrated stoichiometric phases $\mathrm{Ge}_{1} \mathrm{Sb}_{4} \mathrm{Te}_{7}, \mathrm{Ge}_{1} \mathrm{Sb}_{2} \mathrm{Te}_{4}, \mathrm{Ge}_{2} \mathrm{Sb}_{2} \mathrm{Te}_{5}$ and one as a metastable single-phase compound around $\mathrm{Ge}_{4} \mathrm{Sb}_{1} \mathrm{Te}_{5}{ }^{25}$. For these compositions we can extract, from our data, the corresponding values of $\mathrm{T}_{\mathrm{C}}$ : $\mathrm{Ge}_{1} \mathrm{Sb}_{4} \mathrm{Te}_{7}-121 .{ }^{\circ} \mathrm{C}, \mathrm{Ge}_{1} \mathrm{Sb}_{2} \mathrm{Te}_{4}-136.5{ }^{\circ} \mathrm{C}, \mathrm{Ge}_{2} \mathrm{Sb}_{2} \mathrm{Te}_{5}-146.2{ }^{\circ} \mathrm{C}$ and $\mathrm{Ge}_{4} \mathrm{Sb}_{1} \mathrm{Te}_{5}-169 .{ }^{\circ} \mathrm{C}$. These values are all systematically slightly higher than the average published values, however they fall within the range of values reported. Note that small differences in $T_{c}$ can arise from the method of synthesis: sputtered, thermally evaporated or melt /quench materials have all been used amongst the literature values. Even within the case of sputtering the synthesis conditions used have been found to affect $T_{C}{ }^{26}$. Furthermore, due to the nature of the crystallisation process, the heating rate used slightly influences the measured crystallisation temperature ${ }^{27-28}$.

From the whole dataset of $T_{C}$ presented in Figures 3A the "valley" of lowest $T_{C}$ in fact appears to follow the $\mathrm{Sb}_{2} \mathrm{Te}_{3}-\mathrm{Ge}_{2} \mathrm{Te}_{3}$ pseudo-binary line. Extracting data along this tie line (within \pm 1 at $\%$ of 60 at\% Te) $\mathrm{T}_{\mathrm{C}}$ increases from $125{ }^{\circ} \mathrm{C}$ to $150{ }^{\circ} \mathrm{C}$. This is shown in Figure $5 \mathrm{C}$ (blue points), and corresponds to the first (lowest temperature) observed crystallisation event in the HTOMPT measurement. It is evident, however, that a second transition can be observed in the HTOMPT measurement at a higher temperature (Figure 2C). The temperature associated with the second apparent phase transition is also plotted in Figure 5C (red points): A compositional dependence of this transition in the range $280^{\circ} \mathrm{C}$ to $210^{\circ} \mathrm{C}$ is observed. In order to assign the first and second phase transitions plotted in Figure 5C, one can refer to the compositional dependences of the phases observed in XRD after annealing at various temperatures (Figure 7). At $150^{\circ} \mathrm{C}$ the amorphous phase has crystallised to the cubic GST phase along the $\mathrm{Sb}_{2} \mathrm{Te}_{3}-\mathrm{Ge}_{2} \mathrm{Te}_{3}$ pseudo-binary line: $\mathrm{T}_{\mathrm{C}}$ (Figure $5 \mathrm{C}$, blue points) is therefore associated with the amorphous to cubic GST phase transition. In order to 
assign the second observed transition (Figure 5C, red points), it is important to note that the $\mathrm{Sb}_{2} \mathrm{Te}_{3}-$ $\mathrm{Ge}_{2} \mathrm{Te}_{3}$ pseudo binary line delimits compositions (higher Te compositions) which also crystallise Te together with the crystalline GST phases with regions (Iower Te compositions) crystallising to exclusively GST phases (Figure 7). At $200^{\circ} \mathrm{C}$ only the cubic GST is observed, while at $300^{\circ} \mathrm{C}$ the hexagonal GST phase is evident: We therefore assign the second observed transition (Figure 5C, red points) to the GST cubic to GST hexagonal transition. In order to confirm this, and rule out the possibility that small amounts of excess Te are responsible for the second phase transition along the tie line, the HTOMPT result for a slightly Te rich composition $\mathrm{Ge}_{13} \mathrm{Sb}_{23} \mathrm{Te}_{64}$ (identified in Figure $2 \mathrm{~A}$ ) is shown in Figure 5A-B: Three phase transitions are now observed. These are associated with the amorphous to cubic GST transition at $149^{\circ} \mathrm{C}$, the amorphous to hexagonal Te crystallisation at $178^{\circ} \mathrm{C}$, and the cubic GST to hexagonal GST transition at $224^{\circ} \mathrm{C}$. The crystallisation of the two GST phases are at temperatures similar to those observed along the tie line, and the Te crystallisation takes place between these temperatures, as indicated in the temperature dependent phases observed in XRD (Figure 7) which show Te crystallisation before the cubic to hexagonal GST phase transition For $\mathrm{Ge}_{13} \mathrm{Sb}_{23} \mathrm{Te}_{64}$.

For compositions along the $\mathrm{Sb}_{2} \mathrm{Te}_{3}-\mathrm{GeTe}$ tie line, the phase mapping after annealing at $200^{\circ} \mathrm{C}$ (Figure 7) confirms that $T_{C}$ (Figure 4) is associated with the amorphous to the cubic GST phase transition. A clear second transition at $\mathrm{T}_{\mathrm{CH}}$ is observed in HTOMPT (not shown) corresponding to the cubic GST to hexagonal GST phases for compositions $\mathrm{Ge}_{1} \mathrm{Sb}_{4} \mathrm{Te}_{7}\left(\mathrm{~T}_{\mathrm{C}}=122^{\circ} \mathrm{C}, \mathrm{T}_{\mathrm{CH}}=230^{\circ} \mathrm{C}\right)$ and $\mathrm{Ge}_{4} \mathrm{Sb}_{1} \mathrm{Te}_{5}\left(\mathrm{~T}_{\mathrm{C}}=\right.$ $170^{\circ} \mathrm{C}, \mathrm{T}_{\mathrm{CH}}=225^{\circ} \mathrm{C}$ ) in line with expectation ${ }^{19}$. For compositions between these two, towards the middle of the tie line $\left(\mathrm{Ge}_{1} \mathrm{Sb}_{2} \mathrm{Te}_{4}, \mathrm{Ge}_{2} \mathrm{Sb}_{2} \mathrm{Te}_{5}\right)$ the second transition is less distinct and close to the first amorphous/crystalline transition. 
Figure 6 shows the variation of the first transition temperature $T_{c}$ (associated with crystallisation from amorphous to cubic GST) as a function of the tellurium concentration for three different ratios of antimony to germanium. It can be seen that the three ratios present a minimum at around 60 at\% $\mathrm{Te}$, the value of the minimum and the overall data shown is lower for the highest $\mathrm{Sb}$ to Ge ratio and increases as the ratio decreases and that the lowest transition temperature sits in a deeper and narrower range of Te concentration in the case of the lowest $\mathrm{Sb}$ to Ge ratio. These trends in $\mathrm{T}_{\mathrm{C}}$ for crystallisation into the cubic GST structure can be compared to the compositional dependence of crystallisation of GeTe ${ }^{14,} 29-30$. The results of Raoux et al. ${ }^{30}$ (obtained by measuring the drop in resistivity in thin films) and Carria et al. ${ }^{14}$ (obtained from observing changes in reflectivity of thin films) are also plotted in Figure 6, and show a broad minimum in the crystallisation temperature centred at the cubic GeTe compound. These results are consistent with our GST compositional dependencies which also exhibit a broad minimum around 50 at\% Te, with the addition of $\mathrm{Sb}$ to generally lower this minimum, and in addition introduce a narrower minimum in $T_{C}$ which in the limit of zero percent $\mathrm{Sb}$ corresponds to the $\mathrm{Ge}_{2} \mathrm{Te}_{3}$ composition.

X-ray diffraction was performed at room temperature, on the as-deposited library, and on the same library after annealing to $150^{\circ} \mathrm{C}, 200^{\circ} \mathrm{C}, 300^{\circ} \mathrm{C}$ and $400^{\circ} \mathrm{C}$. X-ray diffractograms collected at the various temperatures have been used to understand the crystallographic phase diagram of GST thin films, and to verify the direct correlation between the observed changes in optical contrast (Figure 2) and the extracted values of $T_{C}$ (Figure 1A). This analysis was combined with the use of PDF (Powder Diffraction File $\left.{ }^{\circledR}\right)$ cards from the ICDD (International Centre for Diffraction Data $\left.{ }^{\circledR}\right)$ database to identify the various phases observed. Only the data collected after the annealing to $400^{\circ} \mathrm{C}$ is not shown as some portions of the samples (mainly Te rich compositions) suffered irreversible changes and the XRD data associated with Te loss. The phases identified in the library at the various temperatures are shown in Figure 7. 
Identification of crystalline phases, and mixtures of phases, in thin film combinatorial libraries of solid state materials is a challenge ${ }^{31}$. Preferential orientation or textured growth can lead to missing Bragg reflections and variations in the relative intensities of the observed peaks with respect to conventional powder diffraction. Solid solutions lead to compositionally dependent lattice dimensions which result in shifting diffraction peaks, and long range layered structures over the restricted dimension of a thin film make them difficult to identify. The 529 XRD patterns of the library were background corrected using a single spline curve for the entire dataset. Some attempt was made to use Principle Component Analyses (PCA) and Multivariate Curve Resolution (MCR) to automatically analyse the diffractograms, identify the phases and calculate the relative percentages of each crystal phase for a given composition. However, in an alloy system such as GeSbTe, such approach has its limitations caused by the clustering of many reflections occurring at around a given $2 \theta$ angle, which can be attributed to many different phases. Furthermore, phases exhibiting shifts in peak position due to compositional variations are also difficult to extract and add to the difficulty of accurately identifying a given phase or a mixture of phases. Therefore, results of the PCA-MCR analysis were combined with some manual intervention to extract the phase diagram of the GeSbTe ternary system for the 50 to $100 \mathrm{~nm}$ thick films annealed at $150{ }^{\circ} \mathrm{C}, 200^{\circ} \mathrm{C}$ and $300^{\circ} \mathrm{C}$ shown in Figure 7. The phase diagram of the GST library is best described by five individual components, two known single element phases (Te and $\mathrm{Sb}$ ), the two known stable binaries $\mathrm{GeTe}$ and $\mathrm{Sb}_{2} \mathrm{Te}_{3}$ and a region of solid solution joining the two known binaries $\left(\mathrm{Sb}_{2} \mathrm{Te}_{3}\right.$ and $\left.\mathrm{GeTe}\right)$ in which several metastable compounds have been reported; $\mathrm{Ge}_{1} \mathrm{Sb}_{4} \mathrm{Te}_{7}, \mathrm{GeSb}_{4} \mathrm{Te}_{7}$ and $\mathrm{Ge}_{2} \mathrm{Sb}_{2} \mathrm{Te}_{5}{ }^{32-36}$. A range of additional compounds have also been reported exhibiting long range periodicity ${ }^{37-38}$ which we do not appear to observe in the compositional range of the thin film library. 
The diffractograms obtained from XRD measurement on the as-deposited sample (not show) reveal a library of amorphous materials over the entire compositional spread: the low energy evaporative PVD synthesis with the substrate at low temperature favours the formation of non-equilibrium phases. Lebaudy et al. ${ }^{5}$ reported only relatively small amorphous zones for the GeSbTe system when quenching a bulk sample from melt, but these zones were extended by vapour deposition of thin films. Further studies have shown that amorphous regions have been reported for most of the compositions previously studied and in particular the compositions along the $\mathrm{Sb}_{2} \mathrm{Te}_{3}-\mathrm{GeTe}$ tie-line 39. In additional thin film libraries made using our synthesis method, only compositions close to $\mathrm{Sb}_{2} \mathrm{Te}_{3}$ have been found to be crystalline in the as-deposited state: Slight deviation from this composition and the material was always amorphous.

The results of the crystallographic analysis following annealing to $150{ }^{\circ} \mathrm{C}$ are shown in Figure $7 \mathrm{~A}$. It shows that most of the compositional space remains amorphous and that a region of the GST thin film library has crystallised to mainly the cubic GST phase (Space Group \#225, Fm-3m), with a smaller proportion to the hexagonal GST phase (Space Group \#164, P-3m1). This region of crystallisation is centred primarily along the $\mathrm{Sb}_{2} \mathrm{Te}_{3}-\mathrm{Ge}_{40} \mathrm{Te}_{60}$ tie line. There are also some fields which appear to have both the cubic and hexagonal phases in co-existence. This region of the library exhibiting crystallinity at $150^{\circ} \mathrm{C}$ (Figure $7 \mathrm{~A}$ ) is similar, but slightly broader, than the region where optical changes have been observed in the HTOMPT experiment at $150^{\circ} \mathrm{C}$ (Supplementary Materials). This is also reflected in the compositional dependence of $T_{C}$, which shows a slightly smaller region of materials with a crystallisation temperature below $150^{\circ} \mathrm{C}$ (Figure $\left.2 \mathrm{~A}\right)$. This slight difference in the extent of crystallisation in the library (when comparing $T_{C}$ and XPS) is understandable because of the dynamic nature of the HTOMPT measurement from which $\mathrm{T}_{\mathrm{C}}$ is extracted, the static (long term) annealing in the XRD measurement, and the heating rate dependence of the crystallisation process. In addition, the lower compositional resolution of XRD resulting from the X-ray spot size also broadens 
the boundary between the amorphous and crystalline phases. Nevertheless, the HTOMPT measurement indicates that the first crystallisation along the $\mathrm{Sb}_{2} \mathrm{Te}_{3}-\mathrm{GeTe}$ tie line always occurs below $150^{\circ} \mathrm{C}$ (Figure $5 \mathrm{C}$ ) showing consistency with the XRD phase map at $150^{\circ} \mathrm{C}$ (Figure $7 \mathrm{~A}$ ) and allowing the assignment of this HTOMPT transition to the amorphous to cubic GST phase transition). The cubic GST phase identified in the phase diagrams does not distinguish between the very small variations in the lattice (and hence diffractograms) expected 40 in the rock-salt structure and associated with changes of site occupancies and vacancies ${ }^{41}$.

Annealing the GST library to $200^{\circ} \mathrm{C}$ (Figure 7B) results primarily in two changes: The compositional regions where the cubic GST phase is observed has expanded on both sides of the $\mathrm{Sb}_{2} \mathrm{Te}_{3}-\mathrm{Ge}_{2} \mathrm{Te}_{3}$ tie line, and co-crystallisation of Te is also observed (hexagonal Te, $\mathrm{P}_{3121}$ space group 152) for all compositions which are $\mathrm{Te}$ rich of the $\mathrm{Sb}_{2} \mathrm{Te}_{3}-\mathrm{Ge}_{2} \mathrm{Te}_{3}$ tie line. The latter observation has allowed the association of the phase transition observed in HTOMPT in this Te rich region (Figure $5 \mathrm{~A}-\mathrm{B}$ ) at $178^{\circ} \mathrm{C}$ to the Te crystallisation event. The dominance of the cubic GST phase even at $200^{\circ} \mathrm{C}$ is also consistent with the HTOMPT along the $\mathrm{Sb}_{2} \mathrm{Te}_{3}-\mathrm{Ge}_{2} \mathrm{Te}_{3}$ which indicates that a second phase transition temperature is not observed until temperatures above $200^{\circ} \mathrm{C}$ (Figure $5 \mathrm{C}$ ). Note that the limited compositional regions which exhibited a mixture of cubic and hexagonal GST phases have not changed significantly, although they now observed together with the crystallised Te phase. Also note that the broadening of the compositions exhibiting crystallisation from the amorphous phase at $200^{\circ} \mathrm{C}$ (Figure 7B) correspond reasonably to the $200^{\circ} \mathrm{C}$ contour of $\mathrm{T}_{\mathrm{C}}$ measured in HTOMPT.

Annealing to $300^{\circ} \mathrm{C}$ results in the expansion of the regions of composition where the GST crystalline phases are observed. These consist of the hexagonal phase for Sb rich compositions, mixed cubic and hexagonal at intermediate GeSb compositions, and cubic only for Ge rich compositions. The tie line which now delimits regions of these phases which are observed together with crystalline Te has 
moved slightly towards the $\mathrm{Sb}_{2} \mathrm{Te}_{3}-\mathrm{GeTe}$ tie line. This delimitation of the GST phases coexisting with Te and pure phases is consistent with the phase diagrams of the corresponding SbTe and GeTe systems which also exhibit co-crystallisation of Te for compositions Te rich of the $\mathrm{Sb}_{2} \mathrm{Te}_{3}$ and GeTe phases. Comparison of the $200^{\circ} \mathrm{C}$ and $300^{\circ} \mathrm{C}$ phase diagrams also shows that the cubic to hexagonal phase transition takes place at lowest temperatures for the Sb rich compositions. These results are consistent with the observation that the second phase transition along the $\mathrm{Sb}_{2} \mathrm{Te}_{3}-\mathrm{Ge}_{2} \mathrm{Te}_{3}$ tie line is observed between $200^{\circ} \mathrm{C}$ and $300^{\circ} \mathrm{C}$ (Figure $5 \mathrm{C}$ ) and hence was attributed to the cubic to hexagonal phase transition of GST. The HTOMPT results (Figure $5 \mathrm{C}$ ) also show that the Sb rich compositions chrysalises to the hexagonal phase at the lower temperatures. The only additional phase observed in this library is that of crystallised Sb (space group 166, $\mathrm{R}-3 \mathrm{~m}$ ) for some $\mathrm{Sb}$ rich and Te poor compositions.

Temperatures in excess of $350^{\circ} \mathrm{C}$ have been found to damage Te rich compositions with a notable roughening of the uncapped thin film causing a decrease in optical reflectivity as observed by HTOMPT technique and linked to the observation by SEM of agglomeration of material into lumps. This may be associated with the loss of Te at these temperatures. This renders the analysis of the XRD data quite difficult, nonetheless diffraction patterns attributable to $\mathrm{Ge}$ (space group $227, \mathrm{~F}_{\mathrm{d}-3 \mathrm{~m}}$ ) and GeTe (space group 225, $\mathrm{F}_{\mathrm{m}-3 \mathrm{~m}}$ ) have been identified. However, the full phase diagram at this temperature cannot be drawn with confidence. Since there are large regions in the GST library at $300^{\circ} \mathrm{C}$ which exhibit mixtures of the cubic and hexagonal phases, it is likely that the thin film system does not represent a fully equilibrated system. This is due either because of a slower kinetics in the crystalline to crystalline phase transition, or inhomogeneity of the thin film system associated with interfacial interactions (pinning). Since we can clearly identify in HTOMPT a phase transition associated with the cubic to hexagonal phase transition (Figure 2 and Figure 5C) in regions of compositions where the cubic and hexagonal phases can be in co-existence (Figure 7C), we favour 
the latter explanation for the apparent non-equilibrium of the thin film. Nevertheless, the phase diagram at $300^{\circ} \mathrm{C}$ (Figure $7 \mathrm{C}$ ) is in reasonable agreement with those obtained from bulk samples. The main differences with respect to the work of Labaudy et al ${ }^{5}$ is that we are not able to confirm the existence of the $\mathrm{Sb}_{2} \mathrm{Te}_{3}+\mathrm{GeTe}+\mathrm{Ge}$ and $\mathrm{Sb}_{2} \mathrm{Te}_{3}+\mathrm{Sb}+\mathrm{Ge}$ mixed phases reported for compositions close to the $\mathrm{Sb}_{2} \mathrm{Te}_{3}$ because the library do not cover this region. Legendre et al. ${ }^{42}$ have drawn several isothermal sections of the GST system, focussing on the GeTe-Sb $2 \mathrm{Te}_{3}-\mathrm{Te}$ corner, and our data at $300{ }^{\circ} \mathrm{C}$ is in good agreement with their section at $328^{\circ} \mathrm{C}$.

The electrical sheet resistance was measured at room temperature by 4-point probe, on the as deposited (amorphous) library, and after annealing to $200^{\circ} \mathrm{C}, 300^{\circ} \mathrm{C}$ and $400^{\circ} \mathrm{C}$. The resistivity $(\rho)$ was calculated by linking the sheet resistance to the measured film thicknesses. Due to the extreme spread in the absolute values of $\sigma$ exhibited across the compositional spread of the library, the ternary plots shown in Figure 8 are of $\log (\rho)$ in order to highlight the trends more clearly. Due to irreversible morphological changes that have taken place during the annealing to $400^{\circ} \mathrm{C}$, some caution should be taken when drawing conclusions involving the resistivity measurements from the library annealed at $400^{\circ} \mathrm{C}$ in the Te compositionally rich regions.

The resistivity $\rho$ of the as-deposited amorphous library (Figure $8 \mathrm{~A}$ ) are in the range $10<\rho<10^{5}$ Ohms- $\square$ and show a variation linked primarily with the Te concentration: The Te rich compositions exhibit the largest values of $\rho$. For a given Te concentration, $\rho$ is found to be higher for Ge rich composition, and decreases as the Sb content increases. Overall the lowest values of $\rho$ are found for compositions containing less than 30at\% Te. Annealing the library to $200^{\circ} \mathrm{C}$ (Figure $8 \mathrm{~B}$ ) is accompanied by a large decrease in $\rho$ for the Te rich compositions which can be associated with the crystallisation of the alloys, as shown in the HTOMPT (Figure 2) and the XRD (Figure 7). The Te poor compositions, which exhibit the highest values of $\mathrm{T}_{\mathrm{C}}$ (Figure 2) and remain amorphous at $200^{\circ} \mathrm{C}$ 
(Figure 7B) show very little change in resistivity. The result is that the range of resistivity exhibited by the library after annealing to $200^{\circ} \mathrm{C}$ lies in the range $10^{-4}<\rho /$ Ohms- $\square<1$.

Annealing the library to $300^{\circ} \mathrm{C}$ and then $400^{\circ} \mathrm{C}$ results in an ever-decreasing proportion of the library remaining amorphous (Figure 2 and 9) resulting in a concomitant change in the values of $\rho$ (Figure $8 \mathrm{C}$ and $8 \mathrm{D})$. The changes in the values of $\rho$ of alloys which have already crystallised at $200^{\circ} \mathrm{C}$ do not change significantly during annealing to higher temperature. The range of resistivity values over the compositional spread $\left(10^{-4}<\rho<1\right)$ has not changed at $300^{\circ} \mathrm{C}$. This behaviour is consistent with a sharp decrease in resistivity around the crystallisation temperature, followed by a smaller gradual decrease in resistivity as the temperature is increased further, in agreement with the published data 30,40 .

Annealing the library from $200^{\circ} \mathrm{C}$ to $300^{\circ} \mathrm{C}$ also results in the portions of the sample which have not yet crystallised to exhibit a slight increase in resistivity. This is believed to be due to the "nonequilibrium" nature of the material synthesised during the deposition, and may be associated with the pre-structural ordering (incubation) which has been shown to be a pre-requisite for very highspeed reversible switching ${ }^{43}$. Materials that have been newly sputtered exhibit non-equilibrium behaviour and this results in production phase change memory cells undergo a "burning in" phase, i.e. initialization through cycling ${ }^{44}$. Our resistivity values for the as deposited amorphous phases are larger than those reported in the literature for the room temperature measurement by one order of magnitude, a difference within the variation shown by Sarkar and Gleixner ${ }^{44}$ which can also be explained by the nature of the synthesis method used. As expected, once crystallised the resistivity of selected compositions within the sample are in good agreement with published data. Resistivity values have also been extracted for the four compositions of interest along the $\mathrm{Sb}_{2} \mathrm{Te}_{3}-\mathrm{GeTe}$ tie-line (see Table 1). 
Annealing to $400{ }^{\circ} \mathrm{C}$ results in full crystallisation of the library with the resistivity dropping across the entire sample. The region of lowest resistivity (Figure 8D) coincides closely to the region of lowest crystallisation temperatures (Figure $2 \mathrm{~A}$ ). The Te rich corner of the sample annealed to $400^{\circ} \mathrm{C}$ exhibits a relatively large resistivity, especially when compared to the measurement performed on the sample annealed to $300^{\circ} \mathrm{C}$ : The sample exhibited some irreversible morphological changes for the Te rich corner on annealing to $400^{\circ} \mathrm{C}$ and it is likely that these physical changes are the source of the observed increase in resistivity. As mentioned previously and although not measured quantitatively, the changes seen are probably accompanied by a loss of Te for regions containing excess Te.

An important figure of merit for candidate phase change memory materials for RAM applications is the difference in the resistivity between the amorphous and the crystalline state: The resistivity contrast during the phase change. Figure 9 is a colour map of $\left(\rho_{R T} / \rho_{300}\right)$, a measure of the resistive contrast between the amorphous phase and the crystallised phase after annealing to $300^{\circ} \mathrm{C}$. For the compositions which have crystallised there is between a 5 and 8 order of magnitude change in resistivity, with the largest changes associated with the Te rich compositions. This complete range is sufficient for resistive contrast in electrical data storage applications and holds promise for intermediate states for multilevel single cell memory ${ }^{45}$.

\section{Primary Screening of Phase Change Memory Alloys}

The key figures of merit for the intrinsic property of a material suitable for phase change memory (PCM) applications are archival stability, reflected in the stability of the amorphous phase, the write and erase times, associated with the rate of amorphisation and crystallisation respectively, and the longevity of the reversible switching behaviour, requiring a pure crystalline phase without phase 
separation on repeated melt/quenching to the amorphous phase. Additional refinements are necessary to understand and optimise the switching behaviour in a confined thin film memory node, such as the modification of the re-crystallisation rate in a crystalline matrix or by nucleation at other interfaces (thickness effects). In the case of optical phase change memory applications, the optical contrast between the two states is also a key figure of merit, as is the electrical contrast for electrical memory applications. The set of primary screens described here allows the initial selection of improved materials for these memory applications based on a primary screen of their intrinsic materials behaviours in thin film form.

An indicator of the potential archival stability of the amorphous material, and the power requirement for electrical switching (SET), is given by the temperature of crystallisation $\mathrm{T}_{\mathrm{C}}$. For example, the amorphous phase must be stable for 10 years at about $100^{\circ} \mathrm{C}$ (a typical industry benchmark), and for automotive applications stability over 10 years at $150^{\circ} \mathrm{C}$ is required..$^{46}$ For any class of materials, in the case of homogeneous nucleation, there is a close relationship between Tc and the melting temperature $T_{M}{ }^{47}$ so a measure of Tc also reflects on the temperature and hence power requirement for melt/quenching (RESET) in phase change memory applications. In addition, the resistivity of the amorphous and crystalline phases determines the voltage/current characteristics of the SET and RESET operations, which determine energy consumption important in portable applications and "green" data storage. ${ }^{48}$ The resistive contrast between the amorphous and crystalline state allows the state of the memory to be distinguished in the READ operation. Reliable and fast phase change materials suitable for memory applications are also characterised by systems that do not allow phase separation, i.e. compositional segregation during crystallisation from the amorphous phase. The high throughput phase mapping (Figure 7) allows such regions to be identified effectively. In the selection of potential candidate compositions of GST for the PCM applications, it is therefore important to consider a number of figures of merit from the primary screen. For both optical based and PCRAM 
memory applications, the compositional zone delineating regions which co-crystallise Te are clearly delineated, and correspond to compositions which are Te rich with respect to the $\mathrm{Sb}_{2} \mathrm{Te}_{3}-\mathrm{GeTe}$ lie line (Figure 7). The incumbent optical PCM material is $\mathrm{Ge}_{2} \mathrm{Te}_{2} \mathrm{Sb}_{5}$, and hence sits at this boundary. Slight deviations in Te composition could therefore significantly influence its performance. There are more Ge rich compositions on the tie line with potentially higher optical contrast, but these are characterised by a larger Tc. Te poor compositions can also exhibit large optical contrast with the advantage that they are better separated from materials that can co-crystallise Te, but again the value of $T_{C}$ is higher.

In the case of PCRAM applications, the region of most interest is again delineated by the $\mathrm{Sb}_{2} \mathrm{Te}_{3}-$ GeTe tie line. The Te poor compositions do not co-crystallise Te, and there is a large compositional space which is characterised by the two GST crystalline phases (Figure 7). There is also a substantial region of these compositions that exhibit low crystallisation temperatures (Figure 2A). There are, however, a smaller subset of compositions which lie close to the tie line which also exhibit a large dynamic range of resistance change on switching (Figure 9): Most compositions exhibiting a high dynamic range are in the Te rich compositions exhibiting co-crystallisation of Te. The voltage/current switching characteristics of the amorphous and crystalline materials can further be extracted from Figure $8 \mathrm{~A}$ and Figure 8D respectively.

\section{AUTHOR INFORMATION}

\section{Corresponding Author}

*E-mail: beh@soton.ac.uk

\section{ACKNOWLEDGEMENTS}


The support of the UK's Engineering and Physical Science Research Centre is gratefully acknowledged, through research grant EP/G060363/1 entitled "Manufacturing and Application of Next Generation Chalcogenides". We have no underpinning data that needs archival storage within the content of this work.

\section{SUPPORTING INFORMATION}

- Schematic of the HTOMPT apparatus used for monitoring reflectivity changes as a function of temperature and composition.

- Typical diffractograms collected on thin film samples following annealing to $300^{\circ} \mathrm{C}$ and illustrating the phase assignment used to generate the plots of Figures $7 \mathrm{~A}, \mathrm{~B}$ and $\mathrm{C}$ in the main manuscript.

- Movie of reflectivity changes as a function of temperature from room temperature to $300^{\circ} \mathrm{C}$, both in array view and in a ternary plot. 


\section{REFERENCES}

1. Wuttig, M.; Yamada, N. Phase-change materials for rewriteable data storage. Nat. Mater. 2007, 6 (11), 824-832.

2. Lankhorst, M. H. R.; Ketelaars, B.; Wolters, R. A. M. Low-cost and nanoscale non-volatile memory concept for future silicon chips. Nat. Mater. 2005, 4 (4), 347-352.

3. Kyrsta, S.; Cremer, R.; Neuschutz, D.; Laurenzis, M.; Bolivar, P. H.; Kurz, H. Characterization of Ge-Sb-Te thin films deposited using a composition-spread approach. Thin Solid Films 2001, 398, 379-384.

4. Laurenzis, M.; Heinrici, A.; Bolivar, P. H.; Kurz, H.; Krysta, S.; Schneider, J. M. Composition spread analysis of phase-change dynamics in $\mathrm{Ge}_{\mathrm{x}} \mathrm{Sb}_{\mathrm{y}} \mathrm{Te}_{1-\mathrm{x}-\mathrm{y}}$ films embedded in an optical multilayer stack. IEEE Proceedings-Science Measurement and Technology 2004, 151 (6), 394397.

5. Lebaudy, P.; Saiter, J. M.; Grenet, J.; Belhadii, M.; Vautier, C. Identification of amorphous zones in the GeTeSb system. Mater. Sci. Eng., A 1991, 132, 273-276.

6. Mansuripur, M.; Erwin, J. K.; Bletscher, W.; Khulbe, P.; Sadeghi, K.; Xun, X. D.; Gupta, A.; Mendes, S. B. Static tester for characterization of phase-change, dye-polymer, and magnetooptical media for optical data storage. Appl. Opt. 1999, 38 (34), 7095-7104.

7. Ramberg, C. E.; Wang, Y.; Fan, Q.; McDermott, E.; Wang, J.; Kenyon, K.; Field, M.; Hornbostel, M.; Guan, S.; Nguyen, S. Application of high throughout methods to the development of materials for non-magnetic storage. In Advanced Data Storage Materials and Characterization Techniques, Ahner, J. W.; Levy, J.; Hesselink, L.; Mijiritskii, A., Eds. 2004; Vol. 803, pp 271-276.

8. Guerin, S.; Hayden, B. E. Physical vapor deposition method for the high-throughput synthesis of solid-state material libraries. J. Combi. Chem. 2006, 8 (1), 66-73.

9. Liang, R. G.; Peng, C. B.; Nagata, K.; Daly-Flynn, K.; Mansuripur, M. Optical characterization of multilayer stacks used as phase-change media of optical disk data storage. Appl. Opt. 2002, 41 (2), 370-378.

10. Nishimura, K.; Suzuki, M.; Morimoto, I.; Mori, K. Ge-Te-Sb based overwritable phase-change optical disk. Jpn. J. Appl. Phys., Part 1 1989, 28, 135-139.

11. Ohta, T. Phase-change optical memory promotes the DVD optical disk. J. Optoelectron. Adv. Mater. 2001, 3 (3), 609-626.

12. Wuttig, M.; Yamada, N. Phase-change materials for rewriteable data storage. Nat. Mater. 2007, 6 (11), 824-832.

13. Yada, C.; Lee, C. E.; Laughman, D.; Hannah, L.; Iba, H.; Hayden, B. E. A High-Throughput Approach Developing Lithium-Niobium-Tantalum Oxides as Electrolyte/Cathode Interlayers for High-Voltage All-Solid-State Lithium Batteries. J. Electrochem. Soc. 2015, 162 (4), A722-A726.

14. Carria, E.; Mio, A. M.; Gibilisco, S.; Miritello, M.; Bongiorno, C.; Grimaldi, M. G.; Rimini, E. Amorphous-Crystal Phase Transitions in GexTe1-x Alloys. J. Electrochem. Soc. 2012, 159 (2), H130-H139.

15. Gervacio Arciniega, J. J.; Prokhorov, E.; Espinoza Beltran, F. J.; Trapaga, G. Crystallisation of Ge:Sb:Te Thin Films for Pahse Change Memory Application. In Crystallization - Science and Technology, Andreeta, M., Ed. InTech: 2012.

16. Cil, K.; Zhu, Y.; Li, J.; Lam, C. H.; Silva, H. Assisted cubic to hexagonal phase transition in GeSbTe thin films on silicon nitride. Thin Solid Films 2013, 536, 216-219.

17. Marotta, A.; Saiello, S.; Branda, F.; Buri, A. Activation-energy for the crystallization of glass from ddta curves. J. Mater. Sci. 1982, 17 (1), 105-108.

18. Ohta, T.; Inoue, K.; Uchida, M.; Yoshioka, K.; Akiyama, T.; Furukawa, S.; Nagata, K.; Nakamura, S. Phase-change disk media having rapid cooling structure. Jpn. J. Appl. Phys., Part 1 1989, 28, 123-128. 
19. Park, T. J.; Kim, D. H.; Yoon, S. M.; Choi, K. J.; Lee, N. Y.; Yu, B. G.; Choi, S. Y. Phase Transition Characteristics and Device Performance of Sn-doped $\mathrm{Ge}_{2} \mathrm{Sb}_{2} \mathrm{Te}_{5}$ in Phase Change Random Access Memory. Jpn. J. Appl. Phys. 2006, 45 (48), L1273-L1276.

20. Siegel, J.; Schropp, A.; Solis, J.; Afonso, C. N.; Wuttig, M. Rewritable phase-change optical recording in $\mathrm{Ge}_{2} \mathrm{Sb}_{2} \mathrm{Te}_{5}$ films induced by picosecond laser pulses. Appl. Phys. Lett. 2004, 84 (13), 2250-2252.

21. Yamada, N.; Ohno, E.; Nishiuchi, K.; Akahira, N.; Takao, M. Rapid-phase transitions of GeTe$\mathrm{Sb}_{2} \mathrm{Te}_{3}$ pseudobinary amorphous thin-films for an optical disk memory. J. Appl. Phys. 1991, 69 (5), 2849-2856.

22. Reifenberg, J. P.; Panzer, M. A.; Kim, S.; Gibby, A. M.; Zhang, Y.; Wong, S.; Wong, H. S. P.; Pop, E.; Goodson, K. E. Thickness and stoichiometry dependence of the thermal conductivity of GeSbTe films. Appl. Phys. Lett. 2007, 91 (11), 111904

23. Zhang, T.; Song, Z.; Liu, B.; Feng, G.; Feng, S.; Chen, B. Effect of structural transformation on the electrical properties for $\mathrm{Ge}_{1} \mathrm{Sb}_{2} \mathrm{Te}_{4}$ thin film. Thin Solid Films 2007, 516 (1), $42-46$.

24. Friedrich, I.; Weidenhof, V.; Njoroge, W.; Franz, P.; Wuttig, M. Structural transformations of $\mathrm{Ge}_{2} \mathrm{Sb}_{2} \mathrm{Te}_{5}$ films studied by electrical resistance measurements. J. Appl. Phys. 2000, 87 (9), 41304134.

25. Coombs, J. H.; Jongenelis, A.; Vanesspiekman, W.; Jacobs, B. A. J. Laser-induced crystallization phenomena in GeTe-based alloys .2. Composition dependence of nucleation and growth. $J$. Appl. Phys. 1995, 78 (8), 4918-4928.

26. Dieker, H.; Wuttig, M. Influence of deposition parameters on the properties of sputtered $\mathrm{Ge}_{2} \mathrm{Sb}_{2} \mathrm{Te}_{5}$ films. Thin Solid Films 2005, 478 (1-2), 248-251.

27. Dahshan, A. Thermal stability and crystallization kinetics of new As-Ge-Se-Sb glasses. J. NonCryst. Solids 2008, 354 (26), 3034-3039.

28. Morales-Sanchez, E.; Lain, B.; Prokhorov, E.; Hernandez-Landaverde, M. A.; Trapaga, G.; Gonzalez-Hernandez, J. Crystallization process in $\mathrm{Ge}_{2} \mathrm{Sb}_{2} \mathrm{Te}_{5}$ amorphous films. Vacuum 2010, 84 (7), 877-881.

29. Lu, Y. G.; Song, S. N.; Song, Z. T.; Wu, L. C.; Liu, B.; Feng, S. L.; Guo, X. H. Study on TiÖdoped $\mathrm{Ge}_{2} \mathrm{Te}_{3}$ films for phase-change memory application. J. Phys. D: Appl. Phys. 2011, 44 (14), 145102.

30. Raoux, S.; Munoz, B.; Cheng, H. Y.; Jordan-Sweet, J. L. Phase transitions in Ge-Te phase change materials studied by time-resolved x-ray diffraction. Appl. Phys. Lett. 2009, 95 (14),143118.

31. Takeuchi, I.; Long, C. J.; Famodu, O. O.; Murakami, M.; Hattrick-Simpers, J.; Rubloff, G. W.; Stukowski, M.; Rajan, K. Data management and visualization of x-ray diffraction spectra from thin film ternary composition spreads. Rev. Sci. Instrum. 2005, 76 (6), 1-8.

32. Yamada, N. Origin, secret, and application of the ideal phase-change material GeSbTe. Phys. Status Solidi B 2012, 249 (10), 1837-1842.

33. Jain, A.; Hautier, G.; Moore, C. J.; Ping Ong, S.; Fischer, C. C.; Mueller, T.; Persson, K. A.; Ceder, G. A high-throughput infrastructure for density functional theory calculations. Comput. Mater. Sci. 2011, 50 (8), 2295-2310.

34. Jain, A.; Hautier, G.; Ong, S. P.; Moore, C. J.; Fischer, C. C.; Persson, K. A.; Ceder, G. Formation enthalpies by mixing GGA and GGA + U calculations. Phys. Rev. B 2011, 84 (4), 045115.

35. Ong, S. P.; Jain, A.; Hautier, G.; Kocher, M.; Cholia, S.; Gunter, D.; Bailey, D.; Skinner, D.; Persson, K. A.; Ceder, G. The Materials Project 2011.

36. Ong, S. P.; Wang, L.; Kang, B.; Ceder, G. Li-Fe-P-O-2 phase diagram from first principles calculations. Chem. Mater. 2008, 20 (5), 1798-1807.

37. Kosyakov, V. I.; Shestakov, V. A.; Shelimova, L. E.; Kuznetsov, F. A.; Zemskov, V. S. Topological characterization of the Ge-Sb-Te phase diagram. Inorg. Mater. 2000, 36 (10), 10041017. 
38. Shelimova, L. E.; Karpinskii, O. G.; Kretova, M. A.; Kosyakov, V. I.; Shestakov, V. A.; Zemskov, V. S.; Kuznetsov, F. A. Homologous series of layered tetradymite-like compounds in the Sb-Te and $\mathrm{GeTe}_{\mathrm{Sb}} \mathrm{Se}_{3}$ systems. Inorg. Mater. 2000, 36 (8), 768-775.

39. Yamada, N.; Ohno, E.; Nishiuchi, K.; Akahira, N.; Takao, M. Rapid-phase transitions of GeTe$\mathrm{Sb}_{2} \mathrm{Te}_{3}$ pseudobinary amorphous thin films for an optical disk memory. J. Appl. Phys. 1991, 69 (5), 2849.

40. Morales-Sanchez, E.; Prokhorov, E. F.; Gonzalez-Hernandez, J.; Mendoza-Galvan, A. Structural, electric and kinetic parameters of ternary alloys of GeSbTe. Thin Solid Films 2005, 471 (1-2), 243-247.

41. Matsunaga, T.; Kojima, R.; Yamada, N.; Kifune, K.; Kubota, Y.; Tabata, Y.; Takata, M. Single structure widely distributed in a GeTe- $\mathrm{Sb}_{2} \mathrm{Te}_{3}$ pseudobinary system: A rock salt structure is retained by intrinsically containing an enormous number of vacancies within its crystal. Inorg. Chem. 2006, 45 (5), 2235-2241.

42. Legendre, B.; Chhay, H. C.; Bordas, S.; Clavagueramora, M. T. Phase-diagram of the ternarysystem Ge-Sb-Te .1. The subternary GeTe-Sb $\mathrm{Te}_{3}$-Te. Thermochim. Acta 1984, 78 (1-3), 141-157.

43. Loke, D.; Lee, T. H.; Wang, W. J.; Shi, L. P.; Zhao, R.; Yeo, Y. C.; Chong, T. C.; Elliott, S. R. Breaking the Speed Limits of Phase-Change Memory. Science 2012, 336 (6088), 1566-1569.

44. Sarkar, J.; Gleixner, B. Evolution of phase change memory characteristics with operating cycles: Electrical characterization and physical modeling. Appl. Phys. Lett. 2007, 91 (23), 233506.

45. Raoux, S.; Welnic, W.; Ielmini, D. Phase Change Materials and Their Application to Nonvolatile Memories. Chem. Rev. 2010, 110 (1), 240-267.

46. Raoux, S.; Xiong, F.; Wuttig, M.; Pop, E. Phase change materials and phase change memory. MRS Bull. 2014, 39 (8), 703-710.

47. Okui, N. Relationship between crystallization temperature and melting temperature in crystalline materials. J. Mater. Sci. 1990, 25 (3), 1623-1631.

48. Ielmini, D.; Lacaita, A. L. Phase change materials in non-volatile storage. Mater. Today 2011, 14 (12), 600-607. 


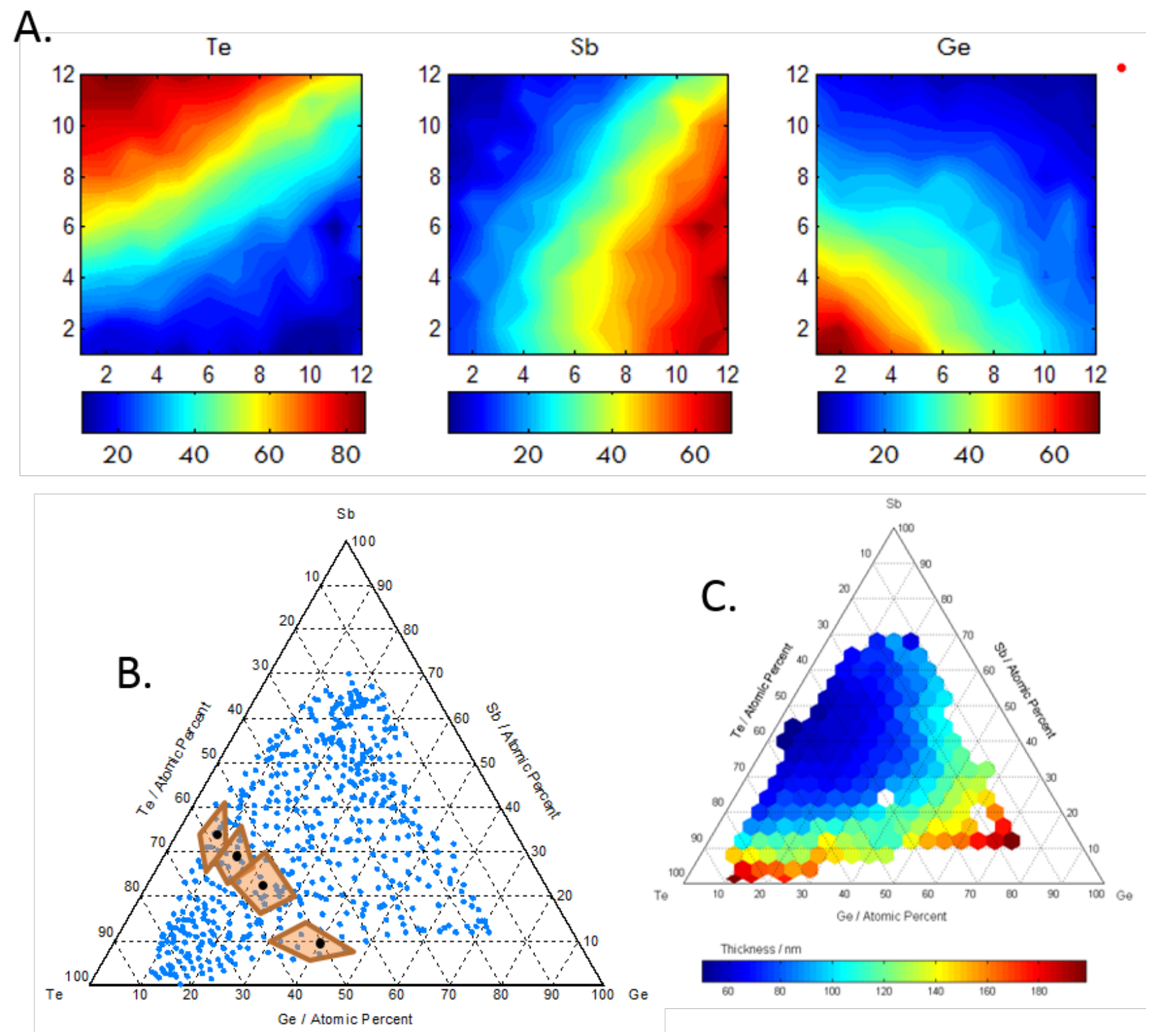

Figure 1

The compositional space covered in a single library of the GeSbTe alloy. a) A colour map of the elemental distribution (atomic percentage) of the elements on the substrate. b) Compositions of the library in the ternary space: 144 compositions are measured by EDX on a 12x12 matrix, and subsequently smoothed and interpolated to 529 points in a $23 \times 23$ matrix or 14400 points in a $120 \times 120$ matrix. The superimposed shaded areas are the regions of compositions covered by Laurenzis et al., ${ }^{4}$ and the four dark points are the $\mathrm{Ge}_{1} \mathrm{Sb}_{4} \mathrm{Te}_{7}, \mathrm{Ge}_{1} \mathrm{Sb}_{2} \mathrm{Te}_{4}, \mathrm{Ge}_{2} \mathrm{Sb}_{2} \mathrm{Te}_{5}$ and $\mathrm{Ge}_{4} \mathrm{Sb}_{1} \mathrm{Te}_{5}$ compositions. c) The thickness ( $\mathrm{nm}$ ) of the GeSbTe alloy thin film library as a function of composition. 

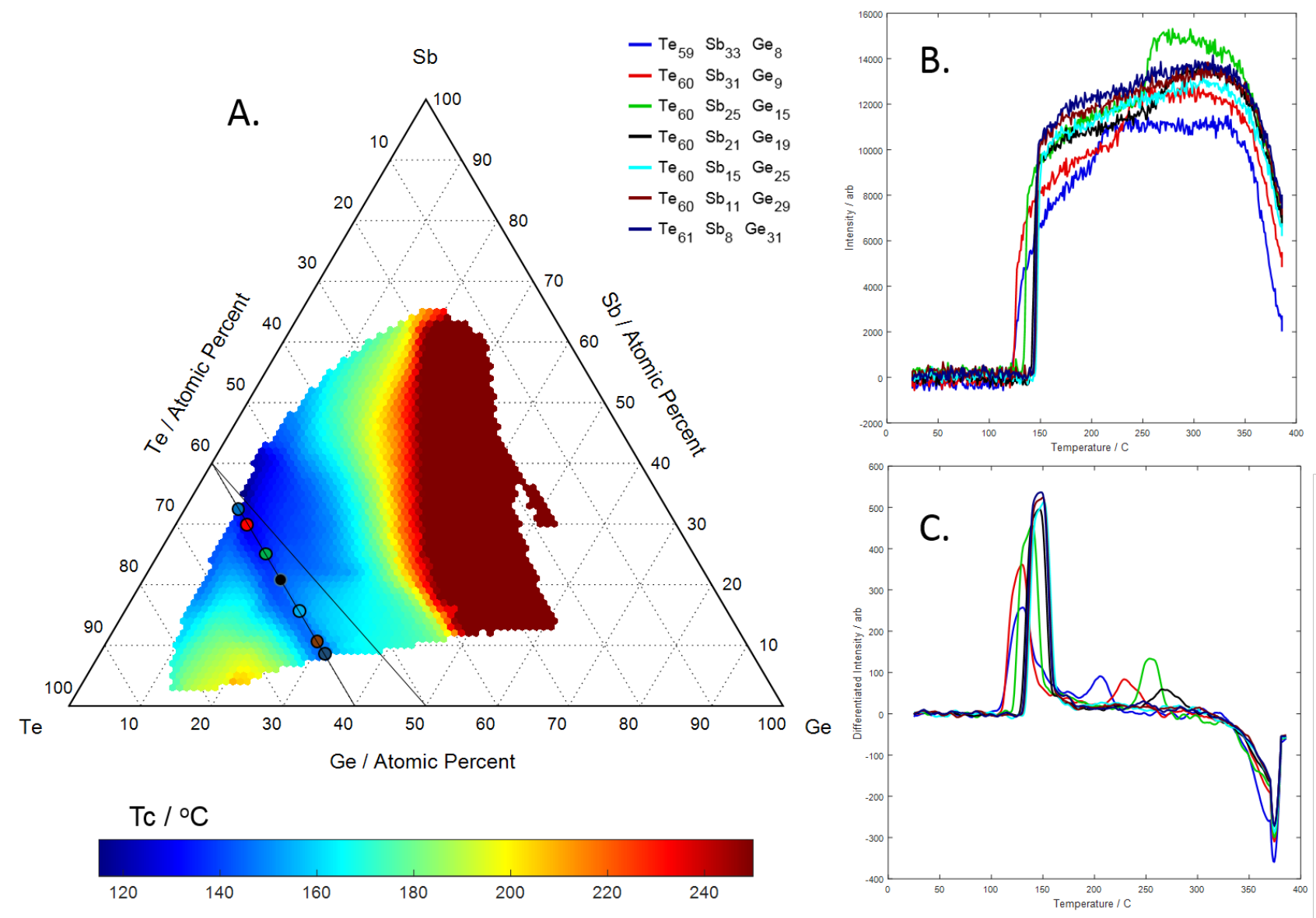

Figure 2

a) The optical reflectivity for selected compositions along the $\mathrm{Sb}_{2} \mathrm{Te}_{3}-\mathrm{Ge}_{2} \mathrm{Te}_{3}$ tie-line of an amorphous GeSbTe library measured as a function of temperature using the HOMPT method. b)The increase in reflectivity is associated with the crystallisation of the amorphous phase. c) The first differential of the temperature dependent reflectivity changes. For some compositions, steps in the change in reflectivity are associated with crystallisation though intermediate structures. 


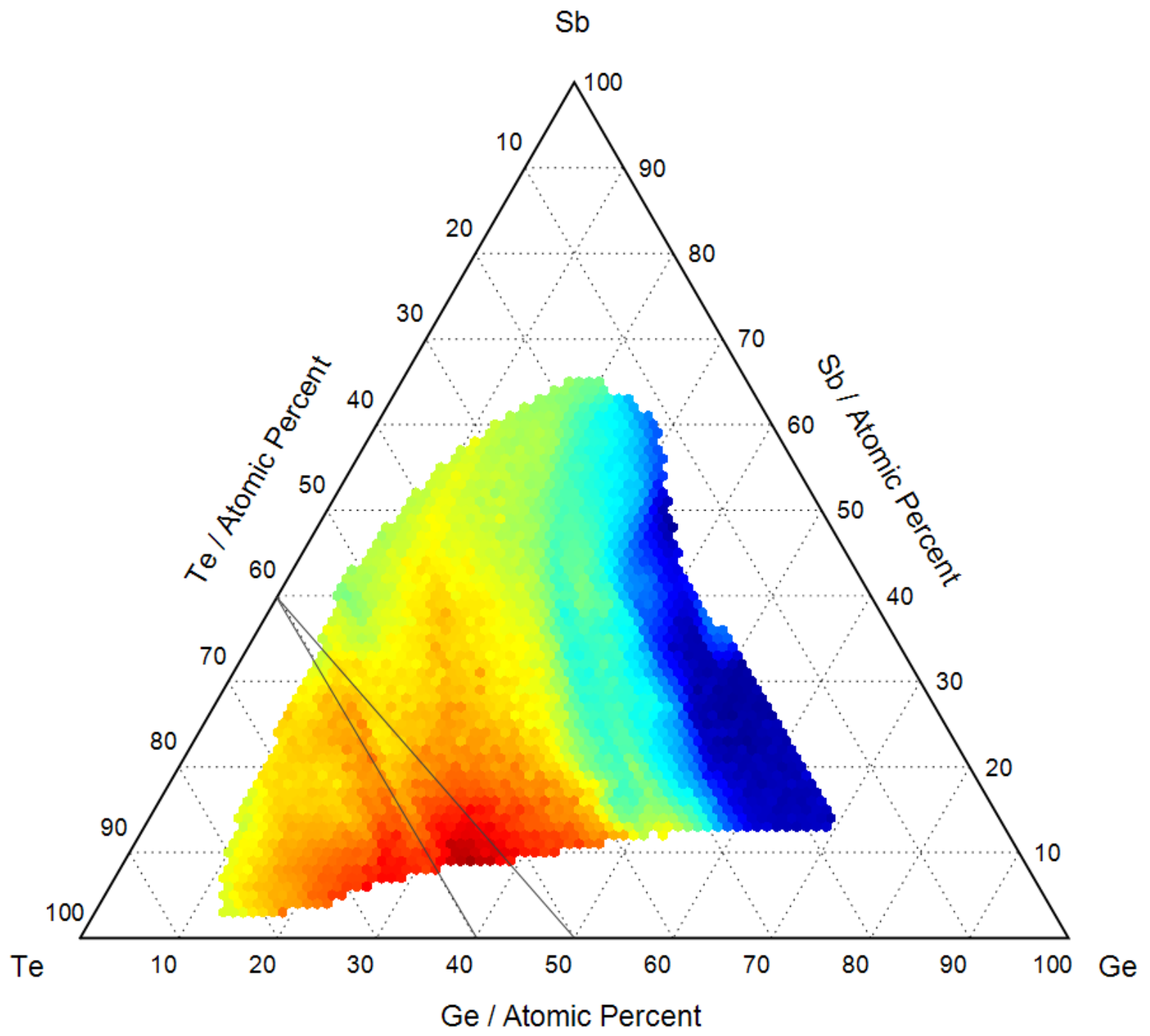

$\%$ change in reflectivity

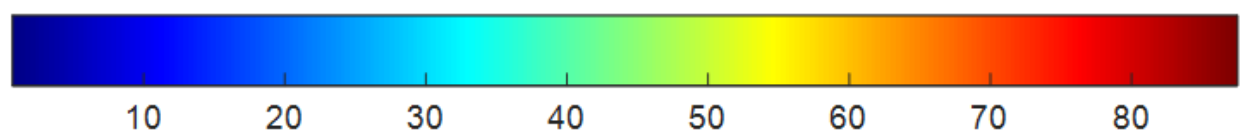

Figure 3

The amorphous to crystalline phase transition for the GeSbTe thin film library measured by HTOMPT, heating between room temperature and $400^{\circ} \mathrm{C}$ showing the change in relative optical reflectivity $\Delta \mathrm{R} / \mathrm{R}$. The black lines represent the position of the know $\mathrm{Sb}_{2} \mathrm{Te}_{3}$-GeTe tie-line as well as the observed valley of lowest crystallisation (Figure $2 \mathrm{~A}$ ) along the $\mathrm{Sb}_{2} \mathrm{Te}_{3}-\mathrm{Ge}_{2} \mathrm{Te}_{3}$ compositions. 


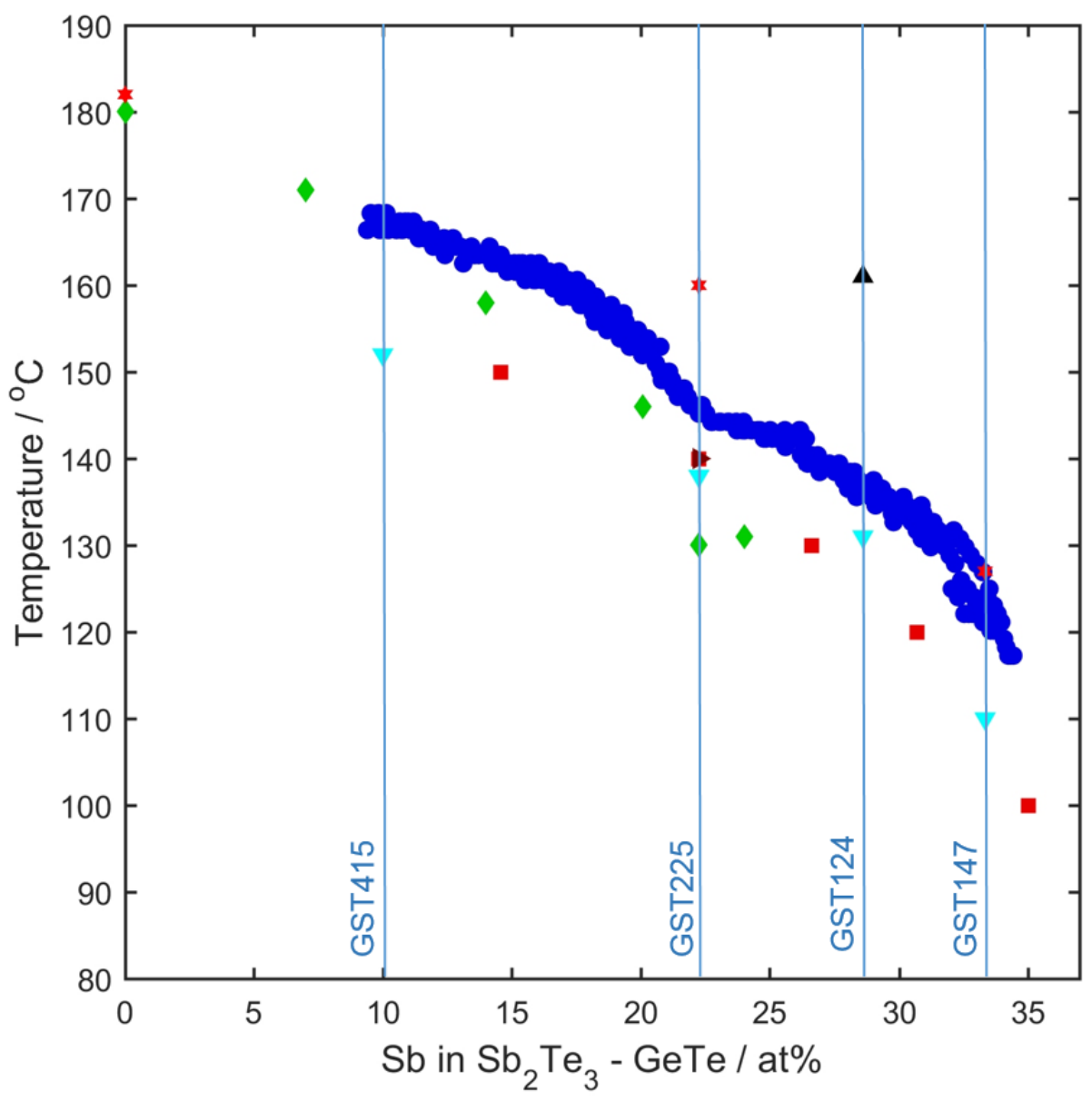

Figure 4

A comparison between our measurement and the literature of the transition temperature between the amorphous and crystalline phases along the $\mathrm{Sb}_{2} \mathrm{Te}_{3}-\mathrm{GeTe}$ tie-line. $\boldsymbol{\bullet}_{\text {this work, }}$ [40], $[22]$, $\boldsymbol{\Delta}_{\text {[23], }} \boldsymbol{\nabla}_{\text {[28], }} \boldsymbol{~}_{\text {[24], }}^{*}$ [2]. 

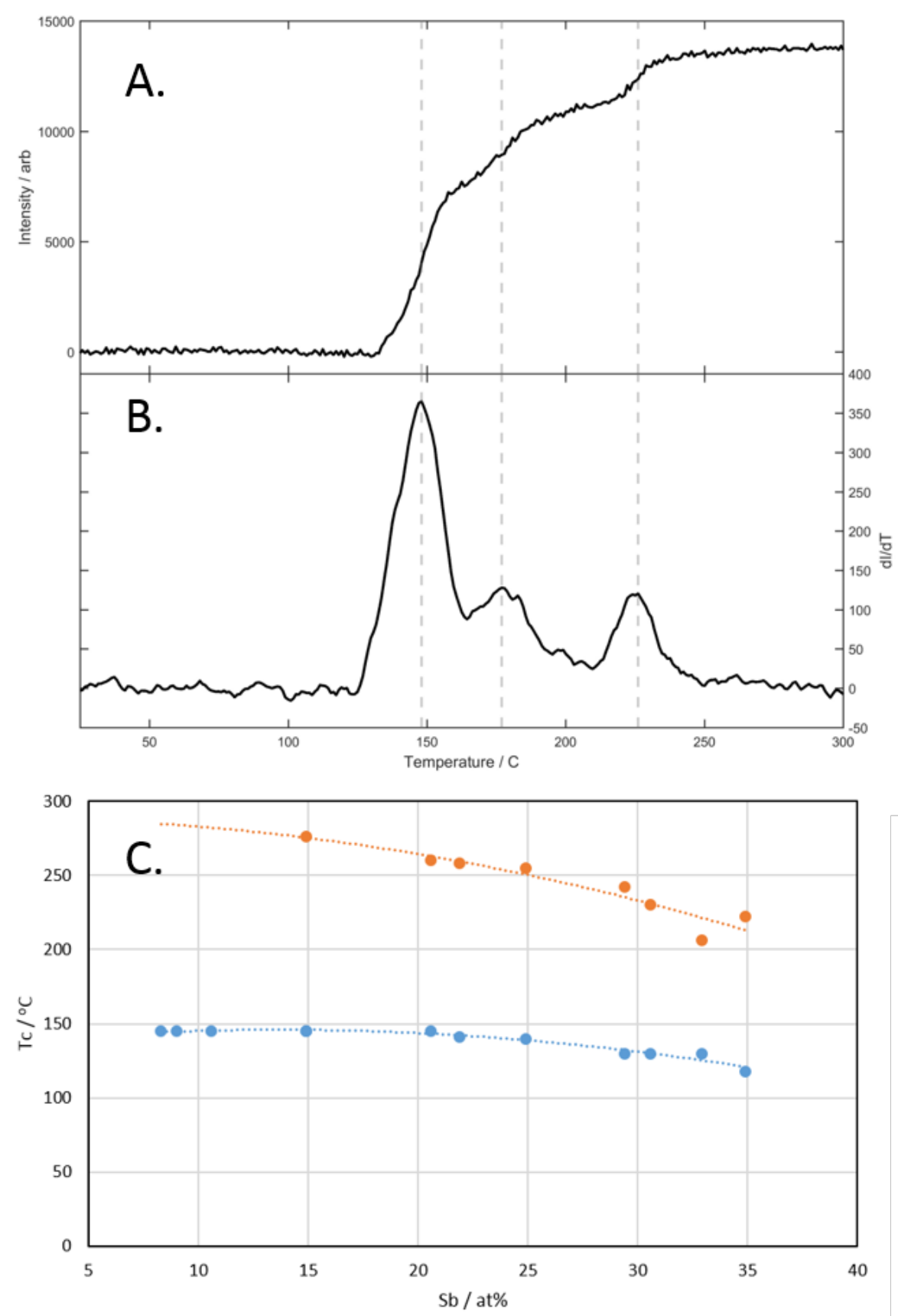

Figure 5

Changes in reflectivity of a GeSbTe alloys as a function of temperature (A.) and associated first differential to determine the temperature of transitions (B.). $\mathrm{Ge}_{12.5} \mathrm{Sb}_{23} \mathrm{Te}_{64.5}$ showing transitions associated with amorphous to cubic GST $\left(149^{\circ} \mathrm{C}\right)$, amorphous to hexagonal Te $\left(178^{\circ} \mathrm{C}\right)$ and cubic GST to hexagonal GST $\left(224^{\circ} \mathrm{C}\right.$ ). Heating rate of $5^{\circ} \mathrm{C} / \mathrm{min}$. (C.) shows the amorphous to crystalline (cubic), blue points, and crystalline (cubic) to crystalline (hexagonal), red points, phase transition along the valley of minimum crystallisation for the $\mathrm{Sb}_{2} \mathrm{Te}_{3}-\mathrm{Ge}_{2} \mathrm{Te}_{3}$ tie-line (Figure 2A). Transition temperatures extracted using the differential approach shown in B. 


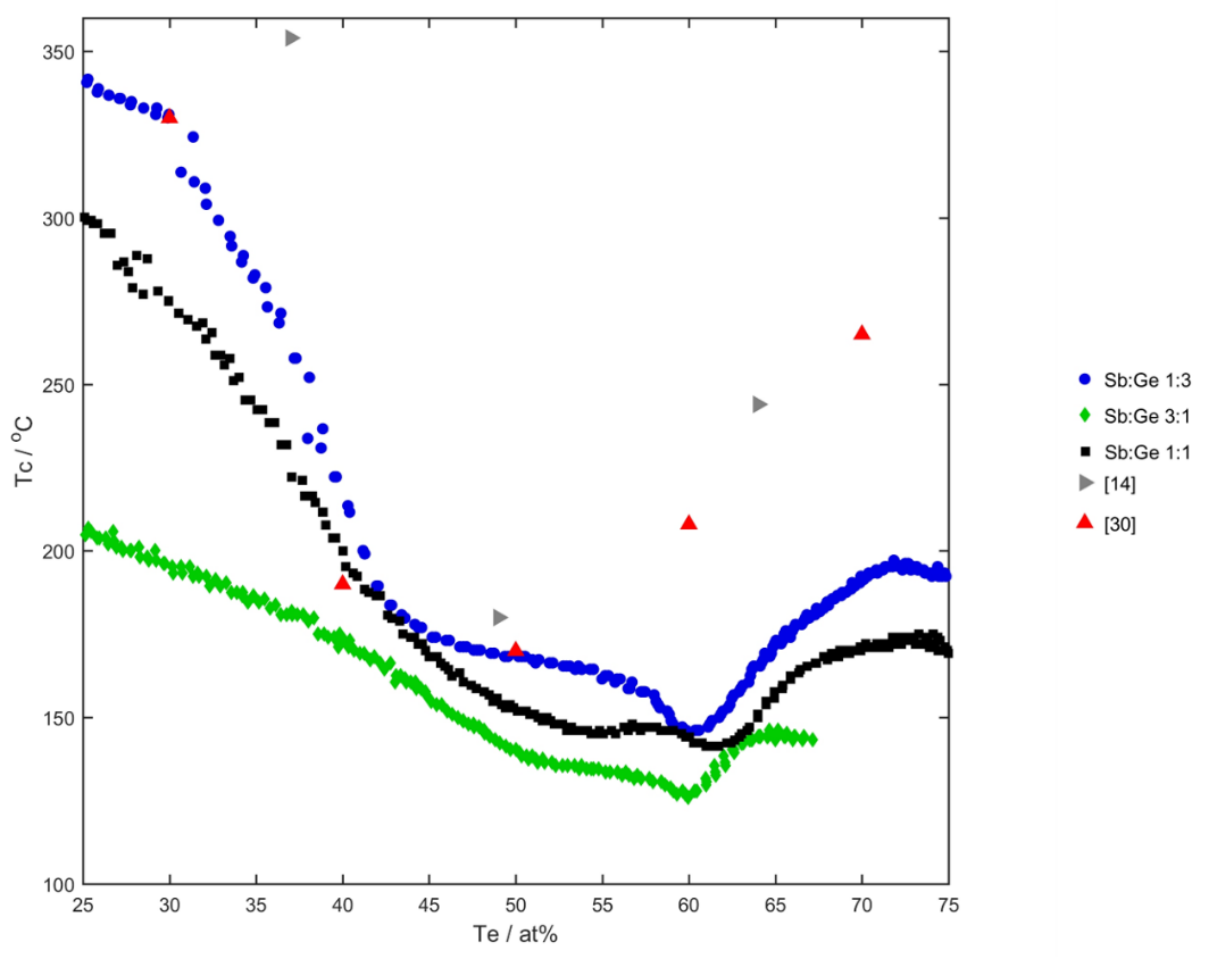

Figure 6

The variation of the first crystallisation transition temperature against Te concentration for different ratio of $\mathrm{Sb}$ to Ge and for literature values of GeTe binary compositions $\nabla^{14}$ and $\Delta 30$. 


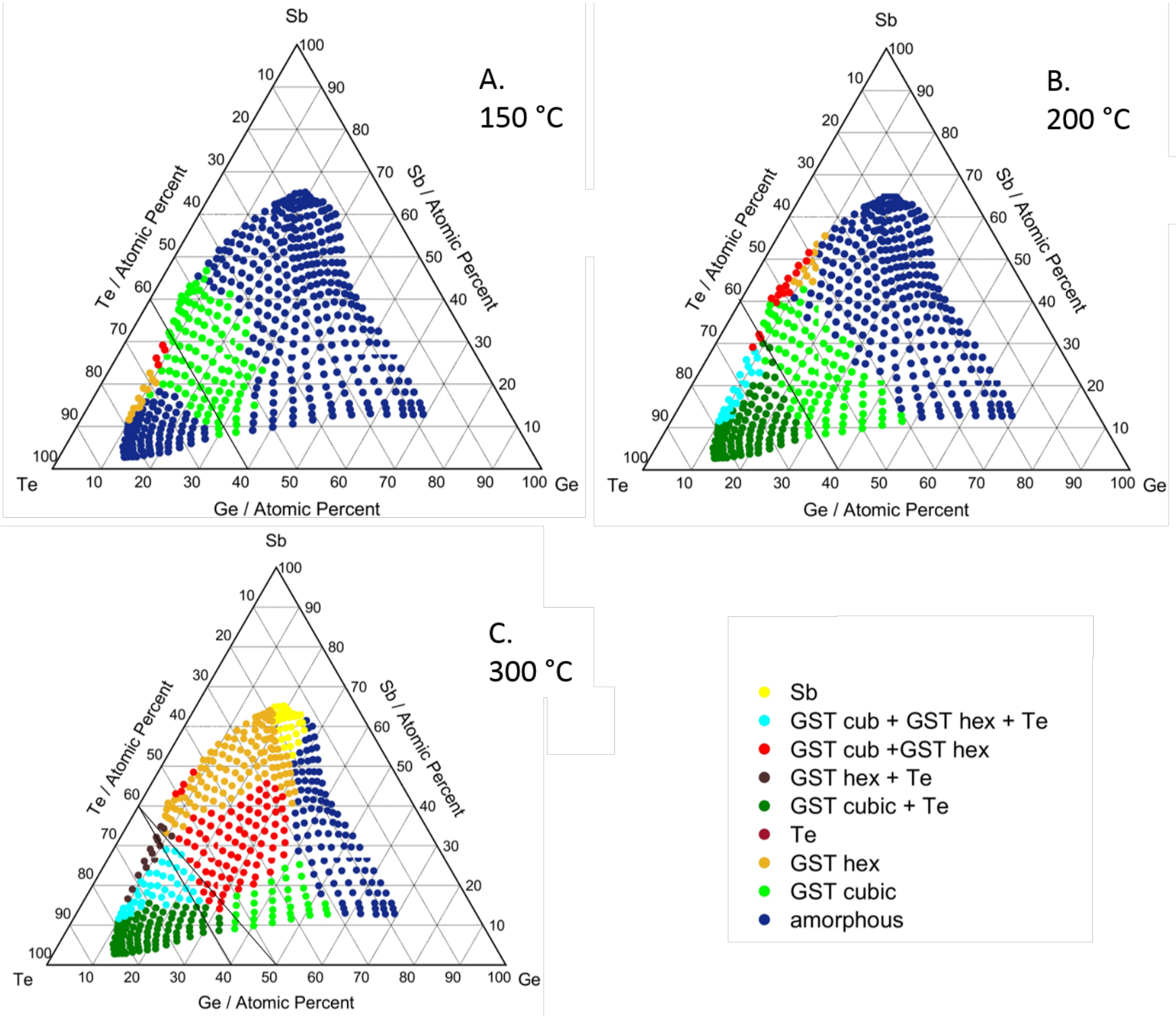

Figure 7

The ternary compositional map of the phases, or mixture of phases, of a GeSbTe thin film library after annealing sequentially to $150{ }^{\circ} \mathrm{C}, 200{ }^{\circ} \mathrm{C}$ and $300^{\circ} \mathrm{C}$. The ternary map of phases present following deposition at $25{ }^{\circ} \mathrm{C}$ showed only the existence of an amorphous phase for the whole compositional range investigated. 


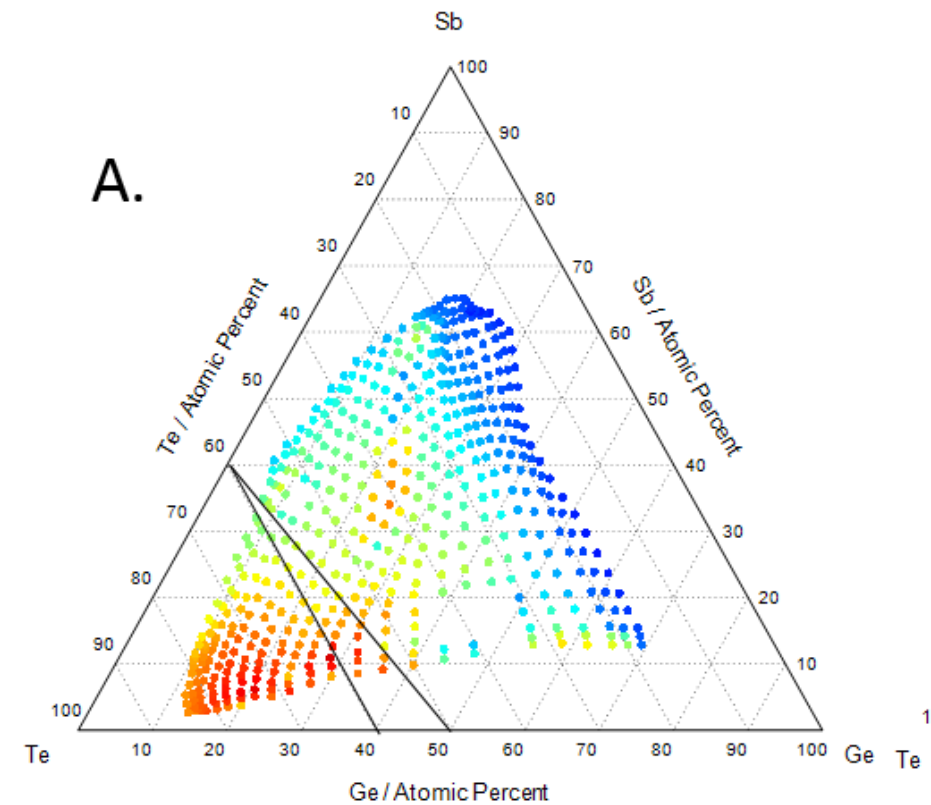

Ge/Atomic Percent

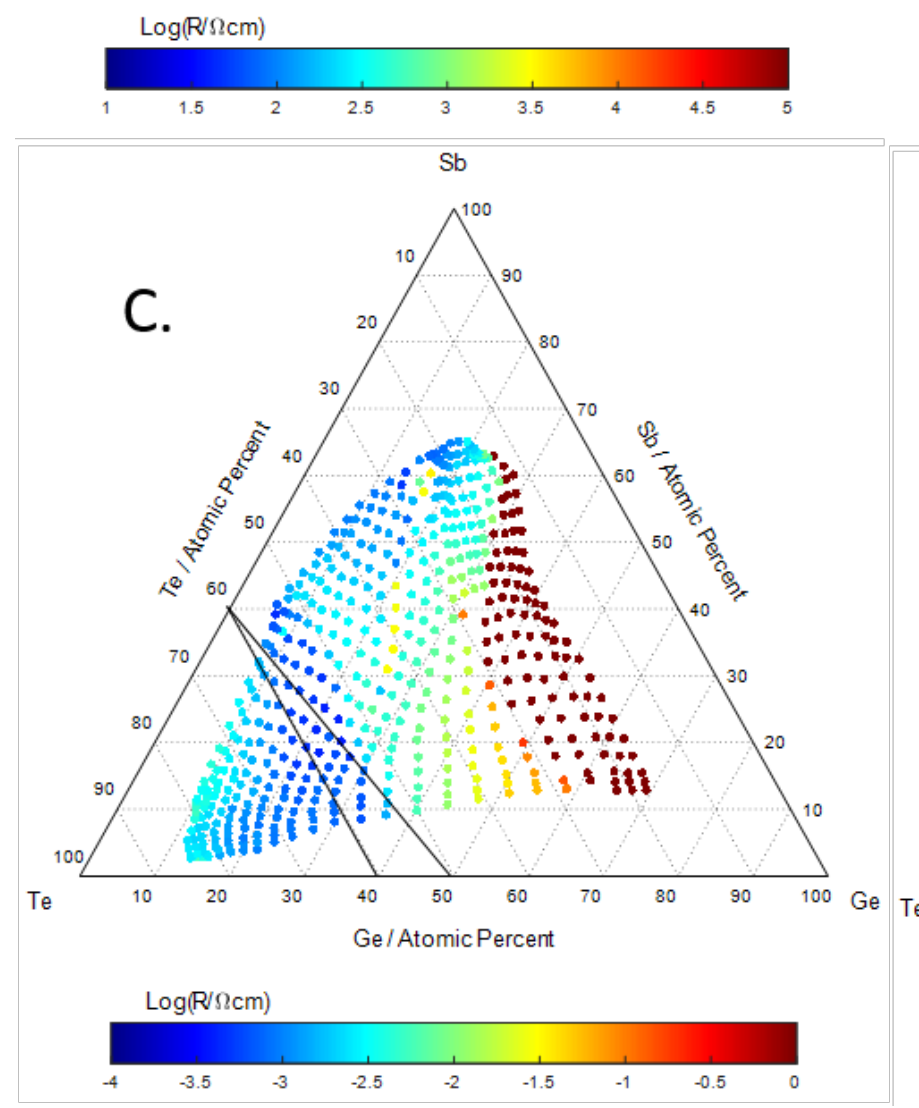

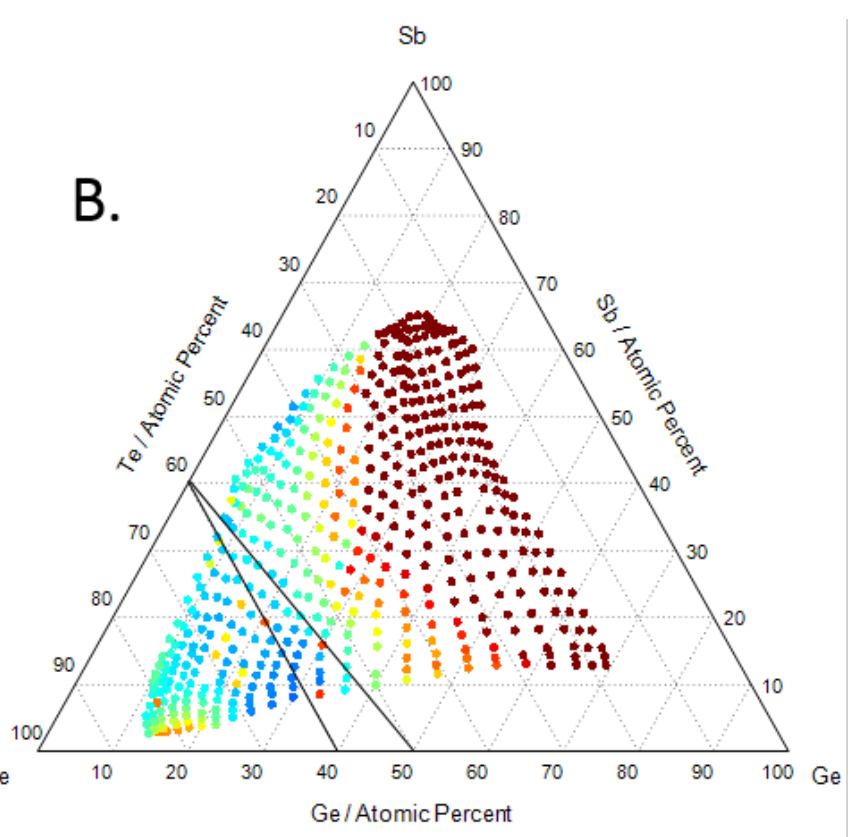

$\log (R / \Omega \mathrm{cm})$
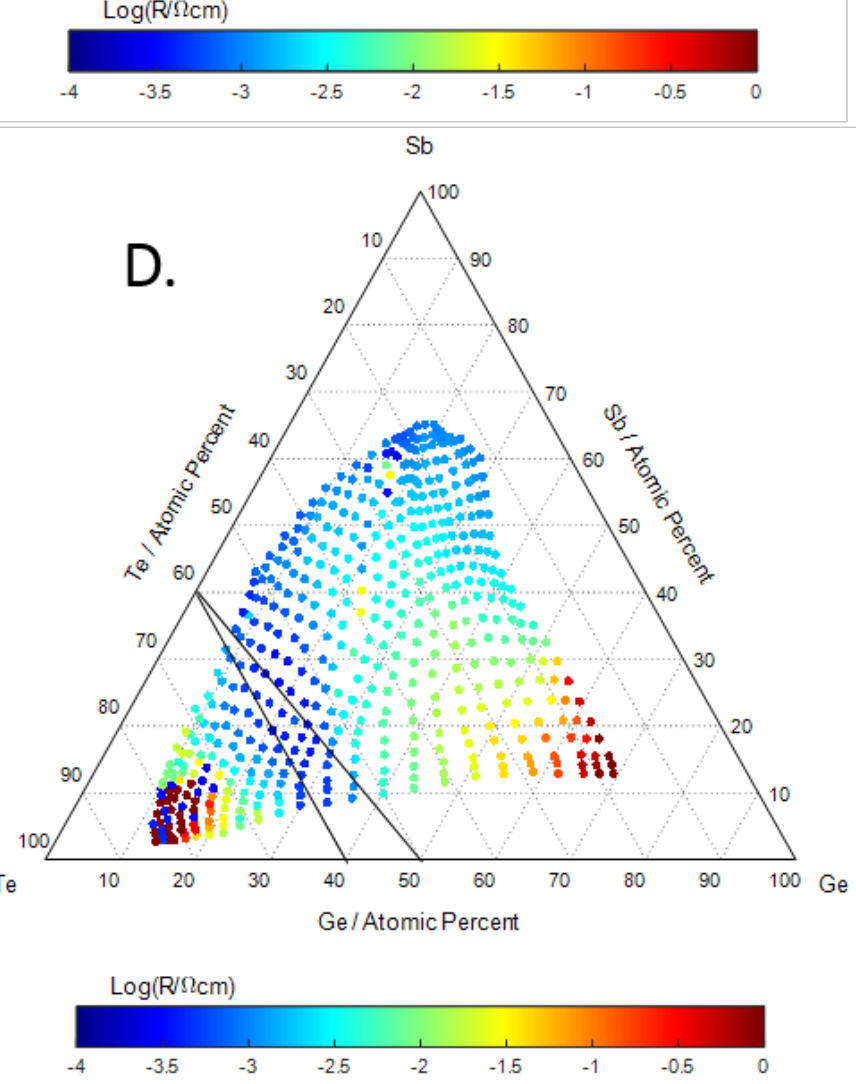

Figure 8

The ternary compositional maps of the $\log \left(R_{e} / \Omega \mathrm{cm}\right)$, where $R_{e}$ is the electrical resistivity measured using a four point probe, for a GeSbTe thin film library: (A) as-deposited (all amorphous), (B) following annealing to $200{ }^{\circ} \mathrm{C},(\mathrm{C})$ following annealing to $300^{\circ} \mathrm{C}$, and (D) following annealing to $400{ }^{\circ} \mathrm{C}$ Data in $\log (\Omega \mathrm{cm})$. Note that the data for the Te rich corner for the library annealed to $400{ }^{\circ} \mathrm{C}$ is affected by the compositional changes of the thin film in this region at this high temperature. 



Figure 9

The ternary compositional map of $\log \left(\mathrm{R}_{\mathrm{RT}} / \mathrm{R}_{300}\right)$, the electrical resistance contrast associated with the amorphous (as deposited) to crystalline (at 300C) phase transition for a GeSbTe thin film library. 


\begin{tabular}{|c|cccc|cccc|}
\cline { 2 - 8 } \multicolumn{1}{c|}{} & Our data / $\Omega c m$ & & & \multicolumn{4}{c|}{ Morales-Sanchez et al.40 / $\Omega \mathrm{cm}$} \\
\hline T / C & GST147 & GST124 & GST225 & GST415 & GST147 & GST124 & GST225 & GST415 \\
\hline 25 & 1001 & 1593 & 2425 & 187 & 130 & 260 & 220 & 291 \\
170 & & & & & $6.50 \mathrm{E}-03$ & $2.50 \mathrm{E}-02$ & $1.50 \mathrm{E}-02$ & $1.78 \mathrm{E}-01$ \\
200 & $6.70 \mathrm{E}-03$ & $9.68 \mathrm{E}-03$ & 0.118 & 0.123 & & & & \\
250 & & & & & $2.20 \mathrm{E}-03$ & $5.30 \mathrm{E}-04$ & $8.60 \mathrm{E}-04$ & $1.90 \mathrm{E}-03$ \\
300 & $6.18 \mathrm{E}-04$ & $3.20 \mathrm{E}-03$ & $4.64 \mathrm{E}-03$ & $2.50 \mathrm{E}-02$ & & & & \\
400 & $5.38 \mathrm{E}-04$ & $6.96 \mathrm{E}-04$ & $3.63 \mathrm{E}-03$ & $1.40 \mathrm{E}-02$ & & & & \\
bulk & & & & & $4.12 \mathrm{E}-04$ & $3.13 \mathrm{E}-04$ & $3.80 \mathrm{E}-04$ & $5.69 \mathrm{E}-04$ \\
\hline
\end{tabular}

Table 1

Comparison of resistivity values for compositions along the $\mathrm{Sb}_{2} \mathrm{Te}_{3}-\mathrm{GeTe}$ tie-line at different temperatures. Our data was obtained at room temperature following annealing to the specified temperatures whereas Morales-Sanchez et al. ${ }^{40}$ report values measured at the specified temperatures 